\title{
Coupled atmosphere-wildland fire modeling with WRF 3.3 and SFIRE 2011
}

\author{
J. Mandel ${ }^{1}$, J. D. Beezley ${ }^{1}$, and A. K. Kochanski ${ }^{2}$ \\ ${ }^{1}$ Department of Mathematical and Statistical Sciences, University of Colorado Denver, Denver, CO, USA \\ ${ }^{2}$ Department of Meteorology, University of Utah, Salt Lake City, UT, USA
}

Received: 5 February 2011 - Published in Geosci. Model Dev. Discuss.: 9 March 2011

Revised: 17 June 2011 - Accepted: 28 June 2011 - Published: 7 July 2011

\begin{abstract}
We describe the physical model, numerical algorithms, and software structure of a model consisting of the Weather Research and Forecasting (WRF) model, coupled with the fire-spread model (SFIRE) module. In every time step, the fire model inputs the surface wind, which drives the fire, and outputs the heat flux from the fire into the atmosphere, which in turn influences the atmosphere. SFIRE is implemented by the level set method, which allows a submesh representation of the burning region and a flexible implementation of various kinds of ignition. The coupled model is capable of running on a cluster faster than real time even with fine resolution in dekameters. It is available as a part of the Open Wildland Fire Modeling (OpenWFM) environment at http://openwfm.org, which contains also utilities for visualization, diagnostics, and data processing, including an extended version of the WRF Preprocessing System (WPS). The SFIRE code with a subset of the features is distributed with WRF 3.3 as WRF-Fire.
\end{abstract}

\section{Introduction}

\subsection{Background}

Wildland fires impact the lives of millions of people and cause major damage every year worldwide, yet they are a natural part of the cycle of nature. Better tools for modeling wildland fire behavior are important for managing fire suppression, planning controlled burns to reduce the fuels, as

Correspondence to: J. Mandel

(jan.mandel@gmail.com) well as to help assess fire danger. Fire models range from tools based on Rothermel (1972) fire spread rate formulas, such as BehavePlus (Andrews, 2007) and FARSITE (Finney, 1998), suitable for operational forecasting, to sophisticated 3-D computational fluid dynamics and combustion simulations suitable for research and analysis, such as FIRETEC (Linn et al., 2002) and WFDS (Mell et al., 2007). BehavePlus, the PC-based successor of the calculator-based BEHAVE, determines the fire spread rate at a single point from fuel and environmental data, FARSITE uses the fire spread rate to provide operation 2-D simulation on a PC, while simulations of wildfires spreading across areas of tens of kilometers performed using FIRETEC or WFDS run slower than real time, even if executed on a parallel supercomputer.

Wildland fire is a complicated multiscale process, from the flame reaction zone on milimeter scale to the synoptic weather scale of hundreds of kilometers. Since direct numerical simulation of wildland fire is computationally intractable and detailed data are not available anyway, compromises in the choice of processes to be modeled, approximations, and parametrizations are essential. Fortunately, a practically important range of wildland fire behavior can be captured by the coupling of a mesoscale weather model with a simple 2-D fire spread model (Clark et al., 1996a,b). Weather has a major influence on wildfire behavior; in particular, wind plays a dominant role in the fire spread. Conversely, the fire influences the atmosphere through the heat and vapor fluxes from burning hydrocarbons and evaporation of fuel moisture. Fire heat output has a major effect on the atmosphere; the buoyancy created by the heat from the fire can cause tornadic strength winds, and the air motion and moisture from the fire can affect the atmosphere away from the fire. It is well known that a large fire "creates its own weather," and

Published by Copernicus Publications on behalf of the European Geosciences Union. 
reproducing qualitatively the general wildland fire shape results from the two-way interaction between the fire and the atmosphere (Clark et al., 1996a,b, 2004; Coen, 2005).

\subsection{Development of the coupled model code}

The coupled WRF and SFIRE code (Mandel et al., 2009) combines the Weather Research and Forecasting Model (WRF) with the ARW dynamical core (Skamarock et al., 2008) with a semi-empirical fire spread model. It is intended to be faster than real time in order to deliver a forecast. The code has grown out of the NCAR's CAWFE code (Clark et al., 1996a,b, 2004; Coen, 2005). CAWFE consists of the Clark-Hall mesoscale atmospheric model, coupled with a tracer-based fire spread model. Although the Clark-Hall model has many good properties, it is a legacy serial code, not supported, and difficult to modify or use for real cases requiring real meteorological data, topography, and fuel maps, while WRF is a parallel supported community code routinely used for real runs. See Coen and Patton (2010) for a further discussion of their relative merits in the wildland fire application. The model was started as WRFFire by Patton and Coen (2004), who proposed a combination of WRF with the tracer-based model from CAWFE, formulated a road map, and made the important observation that the innermost domain of the weather code, which interacts directly with the fire model, needs to run in the Large Eddy Simulation (LES) mode. Patton ported the Fortran 77-based fire module from CAWFE to Fortran 90 and developed the initial coupled code with WRF, with the fire model running on a finer mesh than the atmospheric model, and interpolations between the two. However, instead of using the existing tracer-based CAWFE code, the fire module SFIRE was developed based on the level set method (Osher and Fedkiw, 2003). A more complete timeline is available at http: //www.openwfm.org/wiki/OpenWFM_development_notes.

One of the reasons for replacing the fire propagation scheme was that the representation of the fire region by the level set function was thought to be more flexible than the representation of the burning region in CAWFE by four tracers in each cell of the fire mesh. In particular, the level set function can be manipulated more easily than tracers for the purpose of data assimilation. (Note that the tracers are related to particles, used in computational fluid dynamics, and they represent a Lagrangian approach, while the level set method is an Eulerian approach.) Insertion of the heat fluxes, while fundamentally the same as in CAWFE, had to be redone for WRF variables already in Patton's initial code. Thus, only the code for the calculation of the fire spread rate and the heat fluxes remained from CAWFE.

While SFIRE takes advantage of the experience accumulated with CAWFE, WRF is quite different from the ClarkHall atmospheric model and the fireline propagation algorithm in SFIRE is also different. Thus, it needs to be demonstrated that WRF coupled with SFIRE can deliver similar results as CAWFE, and the coupled model needs to be validated against real fires (Sect. 9).

\subsection{Other related work}

The level set method was used for a surface fire spread model in Mallet et al. (2009). Filippi et al. (2009) coupled the atmospheric model Meso-nh with fire propagation by tracers. Tiger (Mazzoleni and Giannino, 2010) uses a 2-D combusion model based on reaction-convection-diffusion equations and a convection model to emulate the effect of the fire on the wind. FIRESTAR (Morvan and Dupuy, 2004) is a physically accurate wildland fire model in two dimensions, one horizontal and one vertical. UU LES-Fire (Sun et al., 2009) couples the University of Utah's Large Eddy Simulation code with the tracer-based code from CAWFE. See the survey by Sullivan (2009) for a number of other models.

\subsection{Release notes}

SFIRE is public domain software and it has been distributed as WRF-Fire in the WRF source code at http://wrf-model.org since version 3.2, released in April 2010 (Dudhia, 2010). The released version is updated periodically and supported by NCAR. The current version of SFIRE with the latest features and bug fixes and additional visualization tools, guides, and diagnostic utilities are available and supported at http://openwfm.org. WRF-Fire in WRF 3.3 contains a subset of the features described here. In particular, the wind reduction factors and interpolation to different heights for different fuels (Sect. 5) are not included in WRF 3.3, which effectively limits the version of the code there to runs with a single fuel.

This coupled model was briefly treated as one of the topics in Mandel et al. (2009). One of the purposes of this paper is to describe the fire module and the coupling with WRF in the current code in sufficient detail, yet understandable to a reader not familiar with WRF.

New features in since WRF version 3.2 and the paper Mandel et al. (2009) include new ignition models, vertical interpolation of the wind from a logarithmic profile, fetching high-resolution geogrid data, terrain gradient interpolation, and optional input of fuel map, land use map, and topography from files in ideal runs.

\subsection{Contents}

The paper is organized as follows. The model grids are described in Sect. 2. The fire model is presented in Sect. 3, the atmospheric model is briefly reviewed in Sect. 4, and their coupling is described in Sect. 5. Section 6 describes the parallel computing structure of the code and the limitations it implies for the choice of the numerical algorithms. Data input and preprocessing are described in Sect. 7, and the required WRF settings are in Sect. 8. Section 9 contains some early results and a validation plan, and Sect. 10 is the discussion and conclusion. 


\section{Domain, grids, and nodes}

The atmospheric model operates on a logically quadrilateral 3-D grid on the Earth surface, and uses a sequence of horizontally nested grids, called domains. Only the innermost (the finest) atmospheric domain is coupled with the fire model; see also Sect. 8. Scalar variables in the atmospheric model are located at the centers of the 3-D grid cells, while the wind vector components are at a staggered grid at the midpoints of the cell faces. The fire model operates on a refined fire mesh (Fig. 1), and all of its variables are all represented by their values at the centers of the cells of the fire mesh.

\section{Fire model}

The physical fire model is described in Sects. 3.1, 3.2, and 3.3. It consists of functions specifying the fire spread rate and the heat fluxes, and it is essentially the same as a subset of CAWFE (Clark et al., 2004; Coen, 2005). The spread rate calculation is in turn based on BEHAVE (Rothermel, 1972; Andrews, 2007). It is described here in more detail for the sake of reproducibility and to point out the (minor) differences.

The mathematical core of the fire model is described in Sects. 3.4, 3.5, and 3.6. It consists of the numerical algorithms for the fireline propagation, fuel computation, and related functions, such as ignition. The core is independent of any particular fire spread and burn models, and it operates on dimensionless variables. This description here is based on Mandel et al. (2009), with numerous updates and the new developments announced in the introduction.

\subsection{Fuel properties}

Fuel is characterized by the quantities listed in Table 1, which are given at every point of the fire mesh. To simplify the specification of fuel properties, fuels are given as one of 13 Anderson (1982) categories, which are preset vectors of values of the fuel properties. These values are specified in an input text file (namelist.fire), and they can be modified by the user. The user can also specify completely new, custom fuel categories.

The fire spread model is based on average values of fuel properties. The fuel categories assign a single vector of fuel coefficients to a fairly broad description of vegetation cover, with similar average fire propagation properties. In preprocessing, downscaling and upscaling fuel category data to the fire mesh resolution is handled in WPS by setting a cell in the model to whatever category is dominant in the data for that area.

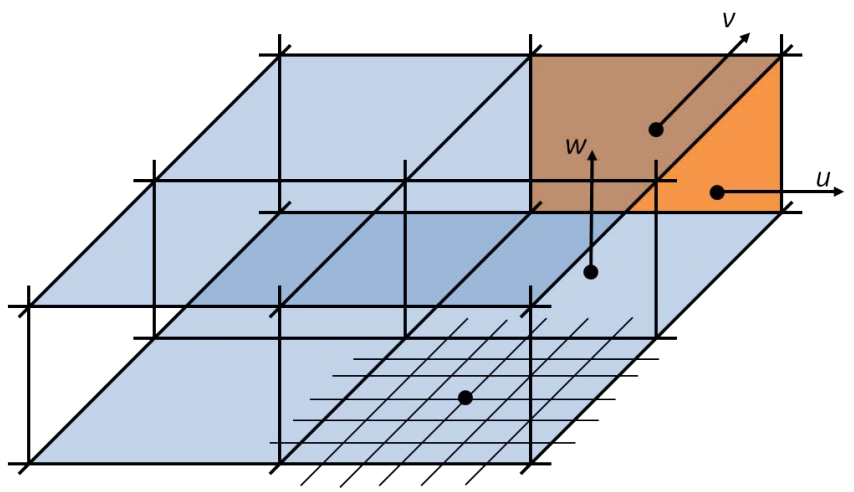

Fig. 1. One $2 \times 2$ tile with the lowest layer of the atmospheric grid and the fire mesh on the surface shown. Wind vector components $u, v, w$ are located at the midpoints of the sides of the atmospheric grid cells. Some faces are colored for perspective.

\subsection{Fire spread rate}

The fire model is posed in the horizontal $(x, y)$ plane the Earth surface is projected on. The semi-empirical approach to fire propagation used here assumes that the fire spread rate is given by the modified Rothermel (1972) formula

$S=R_{0}\left(1+\phi_{\mathrm{W}}+\phi_{\mathrm{S}}\right)$,

where $R_{0}$ is the spread rate in the absence of wind, $\phi_{\mathrm{W}}$ is the wind factor, and $\phi_{\mathrm{S}}$ is the slope factor. The components of Eq. (1) are computed from the fuel properties (Table 1), the wind speed $U$, and the terrain slope $\tan \phi$ following the equations in Table 2. The wind speed $U$ is at the so-called midflame level, which is one of the quantities given in the fuel categories. We support interpolation to $6.1 \mathrm{~m}(21 \mathrm{ft})$ and the use of wind reduction factors (Baughman and Albini, 1980), as well as direct interpolation of the wind to a given midflame level; see Sect. 5.2. We refer to Rothermel (1972) for further details, derivation, and justifications of the computation of the terms in Eq. (1). The only differences here from Rothermel (1972) are the subtraction of the moisture from the fuel load in the computation rather than up front, limiting the slope and the windspeed, and the explicit reduction of wind to midflame height.

The spread rate can be written as

$S=\max \left\{S_{0}, R_{0}+c \min \{e, \max \{0, U\}\}^{b}+d \max \{0, \tan \phi\}^{2}\right\}$

where $S_{0}, R_{0}, b, c, d, e$ are the fuel-dependent coefficients that represent the spread rate internally. These coefficients are stored for every grid point. The representation of the spread rate by Eq. (2) supports also other models, such as a chaparral model from Clark et al. (2004).

At a point on the fireline, denote by $\boldsymbol{n}$ the outside normal to the fire region, $\boldsymbol{U}$ the wind vector, and $z$ the terrain height. The normal component of the wind vector, $U=\boldsymbol{U} \cdot \boldsymbol{n}$, and the 
Table 1. Fuel properties. The notation is from Rothermel (1972) except as indicated. The identifiers are as used in SFIRE or CAWFE. In the input files, some quantities are given in English units per Rothermel (1972); see Wang et al. (2010, p. A-5).

\begin{tabular}{lll}
\hline Symbol & Description & Identifier \\
\hline$a$ & wind adjustment factor (Baughman and Albini, 1980) & windrf \\
& from 6.1 m to the fuel wind ("midflame") height $z_{\mathrm{f}}$ (Sect. 5.2) & \\
$z_{\mathrm{f}}$ & fuel wind height (Sect. 5.2) & fwh \\
$z_{0}$ & fuel roughness height (Sect. 5.2) & $\mathrm{fzo}$ \\
$w$ & fuel weight (i.e., burn time) (s) & \\
& $40 \%$ decrease of fuel in 10 min for $w=1000$ & weight \\
$w_{\ell}$ & total fuel load (kg m ${ }^{-2}$ ) & fgi \\
$\delta_{m}$ & fuel depth (m) & fueldepthm \\
$\sigma$ & fuel particle surface-area-to-volume ratio $\left(1 \mathrm{~m}^{-1}\right)$ & savr \\
$M_{x}$ & moisture content of extinction $(1)$ & fuelmce \\
$\rho_{\mathrm{P}}$ & ovendry fuel particle density ( $\left.\mathrm{kg} \mathrm{m}^{-3}\right)$ & fueldens \\
$S_{\mathrm{T}}$ & fuel particle total mineral content (1) & st \\
$S_{\mathrm{E}}$ & fuel particle effective mineral content (1) & se \\
$h$ & fuel heat contents of dry fuel $\left(\mathrm{J} \mathrm{kg}^{-1}\right)$ & cmbcnst \\
$M_{\mathrm{f}}$ & fuel particle moisture content $(1)$ & fuelmc_g \\
\hline
\end{tabular}

normal component of the terrain gradient, $\tan \phi=\nabla z \cdot \boldsymbol{n}$, are used to determine the spread rate, which is interpreted as the spread rate in the normal direction $\boldsymbol{n}$.

\subsection{Fuel burned and heat released}

Each location starts with fuel fraction $F=1$. Once the fuel is ignited at a time $t_{\mathrm{i}}$, the fuel fraction decreases exponentially,

$F(t)=\exp \left(-\frac{\left(t-t_{\mathrm{i}}\right)}{T_{\mathrm{f}}}\right), \quad t>t_{\mathrm{i}}$,

where $t$ is the time, $t_{\mathrm{i}}$ is the ignition time, $F_{0}$ is the initial amount of fuel, and $T_{\mathrm{f}}$ is the fuel burn time, i.e., the number of seconds for the fuel to burn down to $1 / e \approx 0.3689$ of the original quantity. Since by definition of the fuel weight $w$ (Table 1), the fuel burns down to 0.6 of the original quantity in $600 \mathrm{~s}$ when $w=1000$, we have

$0.6^{\frac{\left(t-t_{\mathrm{i}}\right)}{600} \frac{1000}{w}}=\exp \left(-\frac{\left(t-t_{\mathrm{i}}\right)}{T_{\mathrm{f}}}\right)$

which gives

$T_{\mathrm{f}}=-\frac{600 w}{1000 \ln 0.6} \approx \frac{w}{0.8514}$.

The input coefficient $w$ is used in SFIRE rather than $T_{\mathrm{f}}$ for compatibility with existing fuel models and literature. The fuel weight $w$ is given by the user in the input data as one of the coefficients in the fuel categories. The default values are from the CAWFE code, which, according to Clark et al. (2004, p. 55), were chosen to approximate the mass-loss curve from the BURNUP algorithm (Albini and Reinhardt, 1995). The speed of burning is currently taken to be independent of the wind speed and the fuel moisture. Taking these factors into consideration is a subject of future research, and it will have to be justified by comparison with experiments.

The average sensible heat flux density released in time interval $(t, t+\Delta t)$ is computed as

$\phi_{h}=\frac{F(t)-F(t+\Delta t)}{\Delta t} \frac{1}{1+M_{\mathrm{f}}} w_{\ell} h, \quad\left(\mathrm{~W} \mathrm{~m}^{-2}\right)$

and the average latent heat (i.e., moisture) flux density is given by

$\phi_{q}=\frac{F(t)-F(t+\Delta t)}{\Delta t} \frac{M_{f}+0.56}{1+M_{f}} L w_{\ell}, \quad\left(\mathrm{W} \mathrm{m}^{-2}\right)$

where 0.56 is the estimated mass ratio of the water output from the combustion to the dry fuel, and $L=2.5 \times 10^{6} \mathrm{~J} \mathrm{~kg}^{-1}$ is the specific latent heat of condensation of water at $0^{\circ} \mathrm{C}$, used for nominal conversion of moisture to heat. This computation is from CAWFE.

It should be noted that there is significant uncertainty in the data as well as in the approximations made above, and many factors that influence the spread rate are not accounted for. See Sect. 10.3 for a more complete discussion and possible future improvements.

\subsection{Fire propagation by the level set method}

The model maintains a level set function $\psi$, the time of ignition $t_{\mathrm{i}}$, and the fuel fraction $F$. Denote a point on the surface by $\boldsymbol{x}=(x, y)$. The burning region at time $t$ is represented by a level set function $\psi=\psi(\boldsymbol{x}, t)$ as the set of all points $\boldsymbol{x}$ such that $\psi(\boldsymbol{x}, t) \leq 0$. There is no fire at $\boldsymbol{x}$ if $\psi(\boldsymbol{x}, t)>0$. The fireline is the set of all points $\boldsymbol{x}$ such that $\psi(\boldsymbol{x}, t)=0$. On the fireline, the tangential component of the gradient $\nabla \psi$ is zero. Hence, the outside normal vector at the fireline is

$\boldsymbol{n}=\frac{\nabla \psi}{\|\nabla \psi\|}$. 
Table 2. Computation of the fire spread rate factors in (1) from the fuel properties (Table 1), the wind speed $U$ at $6.1 \mathrm{~m}$, and the terrain slope $\tan \phi$. All equations are from Rothermel (1972) unless otherwise indicated. All input quantities are first converted from metric to English units (BTU-lb-ft-min) to avoid changing the numerous constants in the Rothermel (1972) computations. Further, following CAWFE, the wind is limited to between 0 and $30 \mathrm{~m} \mathrm{~s}^{-1}$ and the slope is limited to nonnegative values. The values marked in bold are the final factors that enter into the spread rate computation (1).

\begin{tabular}{lll}
\hline Equation & Description & Source \\
\hline$R_{0}=\frac{I_{R} \xi}{\rho_{b} \varepsilon Q_{i g}}$ & spread rate without wind & Eq. (52) \\
$\xi=\frac{\exp \left[\left(0.792+0.681 \sigma^{0.5}\right)(\beta+0.1)\right]}{192+0.2595 \sigma}$ & propagating flux ratio & Eq. (42) \\
$I_{\mathrm{R}}=\Gamma w_{n} h \eta_{M} \eta_{s}$ & reaction intensity & Eq. (52) \\
$\eta_{s}=0.174 S_{e}^{-0.19}$ & mineral damping coefficient & Eq. (30) \\
$\eta_{\mathrm{M}}=1-2.59 \frac{M_{f}}{M_{x}}+5.11\left(\frac{M_{f}}{M_{x}}\right)^{2}-3.52\left(\frac{M_{f}}{M_{x}}\right)^{3}$ & moisture damping coefficient & Eq. (29) \\
$w_{\mathrm{n}}=\frac{w_{0}}{1+S_{T}}$ & fuel loading net of minerals & Eq. (24) \\
$w_{0}=\frac{w_{\ell}}{1+M_{f}}$ & total fuel load net of moisture & from CAWFE \\
$\Gamma=\Gamma_{\max }\left(\frac{\beta}{\beta_{\mathrm{op}}}\right)^{A} \exp \left[A\left(1-\frac{\beta}{\beta_{\mathrm{op}}}\right)\right]$ & optimum reaction velocity & Eq. (36) \\
$\Gamma_{\max }=\frac{\sigma^{1.5}}{495+0.594 \sigma^{1.5}}$ & maximum reaction velocity, & Eq. (36) \\
$\beta=\frac{\rho_{b}}{\rho_{\mathrm{P}}}$ & packing ratio & Eq. (31) \\
$\rho_{b}=\frac{w_{0}}{\delta}$ & oven dry bulk density & Eq. (40) \\
$A=\frac{1}{4.77 \sigma^{0.1}-7.27}$ & & Eq. (39) \\
$\varepsilon=\exp \left(-\frac{138}{\sigma}\right)$ & effective heating number & Eq. (14) \\
$Q_{i g}=250 \beta+1116 M_{f}$ & heat of preignition & Eq. (12) \\
$\phi_{W}=C \max U_{a}^{\beta}\left(\frac{\beta}{\beta_{o p}}\right)^{E}$ & wind factor & Eq. (47) \\
$C=7.47 \exp \left(-0.133 \sigma^{0.55}\right)$ & & Eq. (48) \\
$U_{a}=a U$ or $U_{a}=U$ & adjustment to midflame height, if needed & Sect. 5.2 here \\
$E=0.715 \exp \left(-3.59 \times 10^{-4} \sigma\right)$ & & Eq. (50) \\
$\phi_{\mathrm{S}}=5.275 \beta^{-0.3} \tan ^{2} \phi$ & slope factor & Eq. (51) \\
\hline
\end{tabular}

Now consider a point $\boldsymbol{x}(t)$ that moves with the fireline. Then the fire spread rate $S$ at $\boldsymbol{x}$ in the direction of the normal $\boldsymbol{n}$ is

$S=n \cdot \frac{\partial \boldsymbol{x}}{\partial t}$,

and, from the definition of the fireline, $\psi(x(t), t)=0$. By the chain rule and substituting from Eqs. (6) and (7), we have

$$
\begin{aligned}
0 & =\frac{d}{d t} \psi(\boldsymbol{x}, t)=\frac{\partial \psi}{\partial t}+\frac{\partial \psi}{\partial x} \frac{\partial x}{\partial t}+\frac{\partial \psi}{\partial y} \frac{\partial y}{\partial t} \\
& =\frac{\partial \psi}{\partial t}+\|\nabla \psi\|\left(\boldsymbol{n} \cdot \frac{\partial \boldsymbol{x}}{\partial t}\right)=\frac{\partial \psi}{\partial t}+S\|\nabla \psi\| .
\end{aligned}
$$

So, the evolution of the level set function is governed by the partial differential equation $\frac{\partial \psi}{\partial t}+S\|\nabla \psi\|=0$

called the level set equation (Osher and Fedkiw, 2003). The spread rate $S$ is evaluated from (2) for all $\boldsymbol{x}$, not just on the fireline. Since $S \geq 0$, the level set function does not increase with time, and the fire area cannot decrease, which also helps with numerical stability by eliminating oscillations of the level set function $\psi$ in time.

The level set equation is discretized on a rectangular grid with spacing $(\Delta x, \Delta y)$, called the fire grid. The level set function $\psi$ and the ignition time $t_{\mathrm{i}}$ are represented by their values at the centers of the fire grid cells. This is consistent with the fuel data given in the center of each cell also. 
To advance the fire region in time, we use Heun's method (Runge-Kutta method of order 2),

$$
\begin{aligned}
\psi^{n+1 / 2} & =\psi^{n}+\Delta t F\left(\psi^{n}\right) \\
\psi^{n+1} & =\psi^{n}+\Delta t\left(\frac{1}{2} F\left(\psi^{n}\right)+\frac{1}{2} F\left(\psi^{n+1 / 2}\right)\right),
\end{aligned}
$$

The right-hand side $F$ is a discretization of the term $-S\|\nabla \psi\|$ with upwinding and artificial viscosity,

$$
F(\psi)=-S(\boldsymbol{U} \cdot \boldsymbol{n}, \nabla z \cdot \boldsymbol{n})\|\bar{\nabla} \psi\|+\varepsilon \widetilde{\triangle} \psi
$$

where $\boldsymbol{n}=\nabla \psi /\|\nabla \psi\|$ is computed by finite central differences and $\bar{\nabla} \psi=\left[\bar{\nabla}_{x} \psi, \bar{\nabla}_{y} \psi\right]$ is the upwinded finite difference approximation of $\nabla \psi$ by the essentially nonoscillatory (ENO) method of order one (Osher and Fedkiw, 2003, p. 59),

$$
\bar{\nabla}_{x} \psi= \begin{cases}\bar{\nabla}_{x}^{+} \psi & \text { if } \bar{\nabla}_{x}^{-} \psi \leq 0 \text { and } \bar{\nabla}_{x}^{+} \psi \leq 0, \\ \bar{\nabla}_{x}^{-} \psi & \text { if } \bar{\nabla}_{x}^{-} \psi \geq 0 \text { and } \bar{\nabla}_{x}^{+} \psi \geq 0, \\ \bar{\nabla}_{x}^{-} \psi & \text { if } \bar{\nabla}_{x}^{-} \psi \geq 0 \text { and } \bar{\nabla}_{x}^{+} \psi \leq 0, \text { and }\left|\bar{\nabla}_{\mathrm{x}}^{-} \psi\right| \geq\left|\bar{\nabla}_{\mathrm{x}}^{+} \psi\right|, \\ \bar{\nabla}_{x}^{+} \psi & \text { if } \bar{\nabla}_{x}^{-} \psi \geq 0 \text { and } \bar{\nabla}_{x}^{+} \psi \leq 0, \text { and }\left|\bar{\nabla}_{\mathrm{x}}^{-} \psi\right|<\left|\bar{\nabla}_{\mathrm{x}}^{+} \psi\right|, \\ \text { otherwise } 0, & \end{cases}
$$

where $\nabla_{x}^{+} \psi$ and $\nabla_{x}^{-} \psi$ are the right and left one-sided finite differences

$$
\begin{aligned}
& \nabla_{x}^{+} \psi(x, y)=\frac{\psi(x+\Delta x, y)-\psi(x, y)}{\Delta x}, \\
& \nabla_{x}^{-} \psi(x, y)=\frac{\psi(x, y)-\psi(x-\Delta x, y)}{\Delta x},
\end{aligned}
$$

and similarly for $\nabla_{y}^{+} \psi$ and $\nabla_{y}^{-} \psi$. Further, in Eq. (11), $\varepsilon$ is scale-free artificial viscosity $(\varepsilon=0.4$ here $)$, and

$$
\begin{aligned}
\widetilde{\Delta \psi}= & \nabla_{x}^{+} \psi-\nabla_{x}^{-} \psi+\nabla_{y}^{+} \psi-\nabla_{y}^{-} \psi \\
= & \frac{\psi(x+\Delta x, y)-2 \psi(x, y)+\psi(x-\Delta x, y)}{\Delta x} \\
& + \text { similar term for } y
\end{aligned}
$$

is the five-point Laplacian of $\psi$ scaled so that the artificial viscosity is proportional to the mesh step,

$$
\widetilde{\Delta \psi} \approx \Delta x \frac{\partial^{2} \psi}{\partial x^{2}}+\Delta y \frac{\partial^{2} \psi}{\partial y^{2}} .
$$

Although the ENO method theoretically should alone be sufficient to prevent instabilities, we have found that the additional viscosity was needed, otherwise in some cases instabilities do occur.

A numerically stable scheme with upwinding, such as Eq. (12), is required to compute the term $\|\nabla \psi\|$ in the level set Eq. (9). However, in our tests, the gradient by standard central differences,

$$
\begin{aligned}
\nabla \psi \approx & {\left[\frac{\psi(x+\Delta x, y)-\psi(x-\Delta x, y)}{2 \Delta x},\right.} \\
& \left.\frac{\psi(x, y+\Delta y)-\psi(x, y-\Delta y)}{2 \Delta y}\right],
\end{aligned}
$$

worked better in the computation of the normal vector $\boldsymbol{n}$ by Eq. (6), which is used to evaluate the normal component of the wind and the slope in Eq. (2).

We have found that even if the scheme is first-order in space, a second-order method in time was required. For a first-order method (Euler's method), there was too much systematic error in the positive direction, causing the fire to disappear quickly.

Before computing the finite differences up to the boundary, the level set function is extrapolated to one layer of nodes beyond the boundary. However, the extrapolation is not allowed to decrease the value of the level set function to less than the value at either of the points it is extrapolated from. For example, when $(i, j)$ is the last node in the domain in the direction $x$, the extrapolation

$\psi_{i+1, j}=\max \left\{\psi_{i j}+\left(\psi_{i j}-\psi_{i-1, j}\right), \psi_{i j}, \psi_{i-1, j}\right\}$,

is used, and similarly in the other cases. This is needed to avoid numerical instabilities at the boundary. Otherwise, a decrease in $\psi$ at a boundary node, which may happen with non-homogeneous fuels in real data, is amplified by the extrapolation, and $\psi$ keeps decreasing at that boundary node in every time step until it becomes negative, starting a spurious fire.

The model does not support fire crossing the boundary of the domain. When $\psi<0$ is detected near the boundary, the simulation terminates. This is not a limitation in practice, because the fire should be well inside the domain anyway for a proper response of the atmosphere.

The ignition time $t_{\mathrm{i}}$ in the strip that the fire has moved over in one time step is computed by linear interpolation from the level set function. Suppose that the point $\boldsymbol{x}$ is not burning at time $t$ but is burning at time $t+\Delta t$, that is, $\psi(\boldsymbol{x}, t)>0$ and $\psi(\boldsymbol{x}, t+\Delta t) \leq 0$. The ignition time at $\boldsymbol{x}$ satisfies $\psi\left(\boldsymbol{x}, t_{\mathrm{i}}(\boldsymbol{x})\right)=0$. Approximating $\psi$ by a linear function in time, we have

$$
\frac{\psi\left(\boldsymbol{x}, t_{\mathrm{i}}\right)-\psi(\boldsymbol{x}, t)}{t_{\mathrm{i}}(\boldsymbol{x})-t} \approx \frac{\psi(\boldsymbol{x}, t+\Delta t)-\psi\left(\boldsymbol{x}, t_{\mathrm{i}}\right)}{t+\Delta t-t_{\mathrm{i}}(\boldsymbol{x})},
$$

and we take

$$
t_{\mathrm{i}}(\boldsymbol{x})=t+\frac{\psi(\boldsymbol{x}, t) \Delta t}{\psi(\boldsymbol{x}, t)-\psi(\boldsymbol{x}, t+\Delta t)} .
$$

\subsection{Computation of the fuel fraction}

The fuel fraction is approximated over each fire mesh cell $C$ by integrating Eq. (3) over the fire region. Hence, the fuel fraction remaining in cell $C$ at time $t$ is given by

$$
F=1-\frac{1}{\operatorname{area}(C)} \iint_{\substack{\boldsymbol{x} \in C \\ \psi(\boldsymbol{x}, t) \leq 0}} 1-\exp \left(-\frac{t-t_{\mathrm{i}}(\boldsymbol{x})}{T_{\mathrm{f}}(\boldsymbol{x})}\right) d \boldsymbol{x} .
$$

Once the fuel fraction is known, the heat fluxes are computed from Eqs. (4) and (5). This scheme has the advantage 
that the total heat released in the atmosphere over time is exact, regardless of approximations in the computation of the integral Eq. (14). Our objective in the numerical evaluation of Eq. (14) is a method that is second order accurate when the whole cell is on fire, exact when no part of the cell $C$ is on fire (namely, returning the value one), and provides a natural transition between these two cases. Just like standard schemes in numerical analysis can be derived from the requirement that they are exact for all polynomials up to a given degree, the guiding principle here is that the scheme should be exact in as many special cases as possible. Then we expect that the scheme should work well overall.

While the fuel burn time $T_{\mathrm{f}}$ can be interpolated as constant over the whole cell, the level set function $\psi$ and the ignition time $t_{\mathrm{i}}$ must be interpolated more accurately to allow a submesh representation of the burning area and a gradual release of the heat as the fireline moves over the cell. In addition, we need the fuel fraction computed over each mesh cell, because the heat fluxes in the mesh cells are summed up to give the heat flux in an atmospheric cell. Our solution is to split each cell into 4 subcells $C_{j}$, interpolate to the corners of the subcells, and add the integrals,

$$
\begin{aligned}
& \iint_{\substack{\boldsymbol{x} \in C \\
\psi(\boldsymbol{x}) \leq 0}} 1-\exp \left(-\frac{t-t_{\mathrm{i}}(\boldsymbol{x})}{T_{\mathrm{f}}(\boldsymbol{x})}\right) d \boldsymbol{x} \\
& =\sum_{j=1}^{4} \iint_{\substack{x \in C_{j} \\
\psi(\boldsymbol{x}) \leq 0}} 1-\exp \left(-\frac{t-t_{\mathrm{i}}(\boldsymbol{x})}{T_{\mathrm{f}}(\boldsymbol{x})}\right) d \boldsymbol{x},
\end{aligned}
$$

cf., Fig. 2. The level set function $\psi$ is interpolated bilinearly to the vertices of the subcells $C_{j}$, and the burn time $T_{\mathrm{f}}$ is constant on each $C_{j}$, given by its value at the fire grid nodes.

When the whole cell $C$ is on fire (that is, $\psi \leq 0$ on all four vertices of $C), t_{\mathrm{i}}$ is interpolated also linearly to the vertices of the subcells $C_{j}$. However, the case when the fireline crosses the cell $C$ requires a special treatment of the ignition time $t_{\mathrm{i}} ; t_{\mathrm{i}}(\boldsymbol{x})$ has meaningful value only when the node $\boldsymbol{x}$ is on fire, $\psi(\boldsymbol{x}) \leq 0$. Also, on the fireline, $\psi(\boldsymbol{x})=0$ and $t_{\mathrm{i}}(\boldsymbol{x})=t$. Thus, approximating both $\psi$ and $t_{\mathrm{i}}$ in the fire region by linear functions suggests interpolating from the relation

$t_{\mathrm{i}}-t=c \psi$,

for some $c$. We interpolate on the grid lines between two nodes first. If both nodes are on fire, we interpolate $t_{\mathrm{i}}$ bilinearly as before. However, when one cell center is on fire and one not, say $\psi\left(\boldsymbol{a}_{1}\right)>0, \psi\left(\boldsymbol{a}_{2}\right)<0$, we find the proportionality constant $c$ in Eq. (16) from $t_{\mathrm{i}}\left(\boldsymbol{a}_{2}\right)=c \psi\left(\boldsymbol{a}_{2}\right)$, and set $t_{\mathrm{i}}(\boldsymbol{b})=c \psi(\boldsymbol{b})$ at the midpoint $\boldsymbol{b}=\left(\boldsymbol{a}_{1}+\boldsymbol{a}_{2}\right) / 2$. In the case of interpolation to the node $c=\left(a_{1}+a_{2}+a_{3}+a_{4}\right) / 4$ between nodes $\boldsymbol{a}_{1}, \boldsymbol{a}_{2}, \boldsymbol{a}_{3}, \boldsymbol{a}_{4}$, we find the proportionality constant $c$ by solving the least squares problem

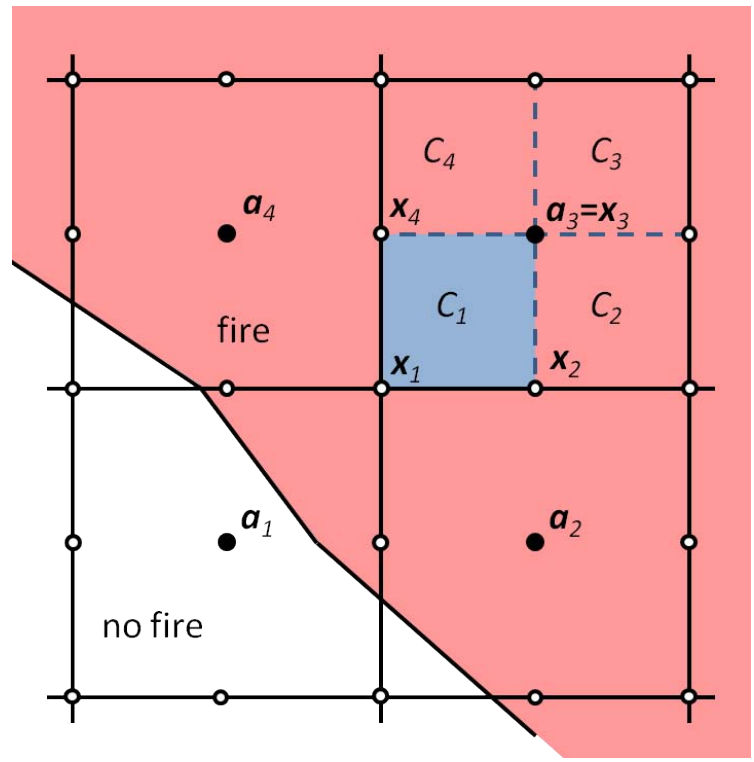

Fig. 2. Division of fire mesh cells into subcells for fuel fraction computation. The level set function $\psi$ and the ignition time $t_{\mathrm{i}}$ are given at the centers $\boldsymbol{a}_{1}, \ldots, \boldsymbol{a}_{4}$ of the cells of the fire grid. The integral (15) over the cell $C$ with the center $\boldsymbol{a}_{3}$ is computed as the sum of integrals over the subcells $C_{1}, \ldots, C_{4}$. While the values of $\psi$ and $t_{\mathrm{i}}$ are known at $\boldsymbol{a}_{3}=\boldsymbol{x}_{3}$, they need to be interpolated to the remaining corners $\boldsymbol{x}_{1}, \boldsymbol{x}_{2}, \boldsymbol{x}_{4}$ of the subcell $C_{1}$ from their values at the points $\boldsymbol{a}_{1}, \ldots, \boldsymbol{a}_{4}$.

$$
\sum_{\substack{j=1 \\ \psi\left(\boldsymbol{a}_{j}\right) \leq 0}}^{4}\left|t_{\mathrm{i}}\left(\boldsymbol{a}_{j}\right)-t-c \psi\left(\boldsymbol{a}_{j}\right)\right|^{2} \rightarrow \min
$$

and set again $t_{\mathrm{i}}(\boldsymbol{c})=c \psi(\boldsymbol{c})$.

To compute the integral over a subcell $C_{j}$, we first estimate the fraction of the subcell that is burning, by

$$
\frac{\operatorname{area}\left\{\boldsymbol{x} \in C_{j}: \psi(\boldsymbol{x}) \leq 0\right\}}{\operatorname{area}\left(C_{j}\right)} \approx \beta=\frac{1}{2}\left(1-\frac{\sum_{k=1}^{4} \psi\left(\boldsymbol{x}_{k}\right)}{\sum_{k=1}^{4}\left|\psi\left(\boldsymbol{x}_{k}\right)\right|}\right),
$$

where $\boldsymbol{x}_{k}$ are the the corners of the subcell $C_{j}$. This approximation is exact when no part of the subcell $C_{j}$, is on fire, that is, all $\psi\left(\boldsymbol{x}_{k}\right) \geq 0$ and at least one $\psi\left(\boldsymbol{x}_{k}\right)>0$; the whole $C_{j}$ is on fire, that is, all $\psi\left(x_{k}\right) \leq 0$ and at least one $\psi\left(x_{k}\right)<0$; or the values $\psi\left(\boldsymbol{x}_{k}\right)$ define a linear function and the fireline crosses the subcell diagonally or it is aligned with one of the coordinate directions.

Next, replace $t_{\mathrm{i}}\left(\boldsymbol{x}_{k}\right)$ by $t$ when $\psi\left(\boldsymbol{x}_{k}\right)>0$ (i.e., the node $\boldsymbol{x}_{k}$ is not on fire), and compute the approximate fraction of the fuel burned as

$$
\begin{aligned}
& \frac{1}{\operatorname{area}(C)} \iint_{\substack{\boldsymbol{x} \in C \\
\psi(\boldsymbol{x}, t) \leq 0}} 1-\exp \left(-\frac{t-t_{\mathrm{i}}(\boldsymbol{x})}{T_{\mathrm{f}}(\boldsymbol{x})}\right) d \boldsymbol{x} \\
& \quad \approx \beta\left(1-\exp \left(-\frac{1}{4} \sum_{k=1}^{4} \frac{t_{\mathrm{i}}\left(\boldsymbol{x}_{k}\right)-t}{T_{\mathrm{f}}}\right)\right)
\end{aligned}
$$


This calculation is accurate asymptotically when the fuel burns slowly and the approximation $\beta$ of the burning area is exact.

\subsection{Ignition}

Typically, a fire starts from a horizontal extent much smaller than the fire mesh cell size, and both point and line ignition need to be supported. The previous ignition mechanism (Mandel et al., 2009) ignited everything within a given distance from the ignition line at once. This distance was required to be at least one or two mesh steps, so that the initial fire is visible on the fire mesh, and the fire propagation algorithm from Sect. 3.4 can catch on. This caused an unrealistically large initial heat flux and the fire started too fast.

The current ignition scheme achieves submesh resolution and zero-size ignition. A small initial fire is superimposed on the regular propagation mechanism, which then takes over. Drip-torch ignition is implemented as a collection of short ignition segments that grows at one end every time step. Multiple ignition segments are also supported.

The model is initialized with no fire by choosing the level set function $\psi\left(\boldsymbol{x}, t_{0}\right)=$ const $>0$. Consider an initial fire that starts at time $t_{\mathrm{g}}$ on a segment $\overline{\boldsymbol{a}, \boldsymbol{b}}$ and propagates in all directions with an initial spread rate $S_{\mathrm{g}}$ until the distance $r_{\mathrm{g}}$ is reached. At the beginning of every time step $t$ such that

$t_{\mathrm{g}} \leq t \leq t_{\mathrm{g}}+\frac{r_{\mathrm{g}}}{S_{\mathrm{g}}}$,

we construct the level set function of the initial fire,

$\psi_{\mathrm{g}}(\boldsymbol{x}, t)=\operatorname{dist}(\boldsymbol{x}, \overline{\boldsymbol{a}, \boldsymbol{b}})-S_{\mathrm{g}}\left(t-t_{\mathrm{g}}\right)$

and replace the level set function of the model by

$\psi(\boldsymbol{x}, t):=\min \left\{\psi(\boldsymbol{x}, t), \psi_{\mathrm{g}}(\boldsymbol{x}, t)\right\}$.

For a drip-torch ignition starting from point $\boldsymbol{a}$ at time $t_{\mathrm{g}}$ at velocity $v$ until time $t_{\mathrm{h}}$, the ignition line at time $t$ is the segment $\overline{\boldsymbol{a}, \boldsymbol{a}+\boldsymbol{v}\left(\min \left\{t, t_{\mathrm{h}}\right\}-t_{\mathrm{g}}\right)}$, and Eq. (19) becomes

$$
\begin{aligned}
\psi_{\mathrm{g}}(\boldsymbol{x}, t)= & \operatorname{dist}\left(\boldsymbol{x}, \overline{\boldsymbol{a}, \boldsymbol{a}+\boldsymbol{v}\left(\min \left\{t, t_{\mathrm{h}}\right\}-t_{\mathrm{g}}\right)}\right) \\
& -\min \left\{r_{\mathrm{g}}, S_{\mathrm{g}}\left(t-t_{\mathrm{g}}\right)\right\}
\end{aligned}
$$

followed again by Eq. (20), at the beginning of every time step begining at time $t$ such that

$t_{\mathrm{g}} \leq t \leq t_{\mathrm{h}}+\frac{r_{\mathrm{g}}}{S_{\mathrm{g}}}$.

The ignition time of newly ignited nodes is set to the arrival time of the fire at the spread rate $S_{\mathrm{g}}$ from the nearest point on the ignition segment.

\section{Atmospheric model}

We summarize some background information about WRFARW from Skamarock et al. (2008), to the extent needed to understand the coupling with the fire module.

The model is formulated in terms of the hydrostatic pressure vertical coordinate $\eta$, scaled and shifted so that $\eta=1$ at the Earth surface and $\eta=0$ at the top of the domain. The governing equations are a system of partial differential equations of the form

$\frac{d \Phi}{d t}=R(\Phi)$,

where $R$ contains also the advection terms, and $\Phi=$ $\left(U, V, W, \phi^{\prime}, \Theta, \mu^{\prime}, Q_{\mathrm{m}}\right)$. The fundamental WRF variables are $\mu=\mu(x, y)$, the hydrostatic component of the pressure differential of dry air between the surface and the top of the domain, written in perturbation form $\mu=\bar{\mu}+\mu^{\prime}$, where $\bar{\mu}$ is a reference value in hydrostatic balance; $U=\mu u$, where $u=u(x, y, \eta)$ is the Cartesian component of the wind velocity in the $x$-direction, and similarly $V$ and $W ; \Theta=\mu \theta$, where $\theta=\theta(x, y, \eta)$ is the potential temperature; $\phi=\phi(x, y, \eta)=$ $\bar{\phi}+\phi^{\prime}$ is the geopotential; and $Q_{\mathrm{m}}=\mu q_{\mathrm{m}}$ is the moisture content of the air. The variables in the state $\Phi$ evolved by Eq. (21) are called prognostic variables. Other variables computed from them, such as the hydrostatic pressure $p$, the thermodynamic temperature $T$, and the height $z$, are called diagnostic variables. The variables that contain $\mu$ are called coupled. The value of the right-hand side $R(\Phi)$ is called tendency. See Skamarock et al. (2008, p. 7-13) for details and the form of $R$.

The system (21) is discretized in time by the explicit 3rd order Runge-Kutta method

$$
\begin{aligned}
\Phi_{1} & =\Phi^{t}+\frac{\Delta t}{3} R\left(\Phi^{t}\right) \\
\Phi_{2} & =\Phi^{t}+\frac{\Delta t}{2} R\left(\Phi_{1}\right) \\
\Phi^{t+\Delta t} & =\Phi^{t}+\Delta t R\left(\Phi_{2}\right)
\end{aligned}
$$

where the differential operator $R$ is discretized by finite differences and the tendencies from physics packages, such as the fire module, are updated only the third Runge-Kutta step (Skamarock et al., 2008, p. 16). In order to avoid small time steps, the tendency in the third Runge-Kutta step also includes the effect of substeps to integrate acoustic modes.

\section{Coupling of the fire and the atmospheric models}

\subsection{Interpolation of the terrain gradient}

The terrain gradient is computed from the terrain height at the best available resolution and interpolated to the fire mesh in preprocessing. If no better resolution is available, the terrain height from the atmospheric model can be used. Interpolating the height and then computing the gradient would cause 
jumps in the gradient, unless high-order interpolation is used, which cause unnatural fire propagation with the fire changing direction at the location of the jumps of the gradient.

\subsection{Wind interpolation and wind reduction factors}

Wind is interpolated horizontally from the atmospheric mesh to the fire mesh, and vertically to a given "midflame" height $z_{\mathrm{f}}$ above the terrain (Baughman and Albini, 1980) by assuming the ideal logarithmic wind profile

$u(z) \approx\left\{\begin{array}{lr}c \ln \frac{z}{z_{0}}, & z \geq z_{0}, \\ 0 & 0 \leq z \leq z_{0},\end{array}\right.$

where $z$ is the height above the terrain, $z_{0}$ is the roughness height, and $c$ is a proportionality constant.

The vertical interpolation algorithm is as follows. For a given horizontal location, denote by $z_{1}, z_{2}, \ldots$ the heights of the atmospheric grid mid-levels and by $u\left(z_{1}\right), u\left(z_{2}\right), \ldots$ the values of the $u$ horizontal wind component at those heights. The heights $z_{k}$ are computed from the geopotential $\phi$, which is a part of the solution in WRF. The horizontal wind components $u$ at $z_{k}$ are then found by horizontal interpolation from the atmospheric mesh, and interpolated vertically to the given height $z_{\mathrm{f}}$ by $\log$-linear interpolation. That is, $u\left(z_{\mathrm{f}}\right)$ is determined by $1-D$ piecewise linear interpolation of the values $u\left(z_{0}\right)=0, u\left(z_{1}\right), u\left(z_{2}\right), \ldots$ at $\ln z_{0}, \ln z_{1}, \ln z_{2}, \ldots$ to $\ln z_{\mathrm{f}}$; if $z_{\mathrm{f}} \leq z_{0}$, we set $u\left(z_{\mathrm{f}}\right)=0$. The $v$ component of the wind is interpolated in the same way. The computation is organized in such way that only the vertical levels that are actually needed are evaluated above every fire mesh node.

Note that the vertical interpolation is exact if the wind profile obeys the logarithmic profile Eq. (23) exactly. This is a desirable property in numerical analysis: for example, linear interpolation can be defined by the fact that it is exact for all linear functions.

A commonly used value for the roughness height is $z_{0}=$ $0.13 H$, where $H$ is the height of the vegetation. The roughness height $z_{0}$ and the height $z_{\mathrm{f}}$ to interpolate to (the "midflame" height) are properties of the fuel category, and they are given by the user in input data. The roughness height is also known from land use in WRF, though that value may not be the same.

We also support the use of the wind reduction factors, following Baughman and Albini (1980). The wind reduction (or adjustment) factor $a$ is used to determine the vertically interpolated wind $u\left(z_{\mathrm{f}}\right)$ from wind measured at a reference height $z_{\text {ref }}(20 \mathrm{ft}=6.096 \mathrm{~m}$ in BEHAVE), by assuming the ideal logarithmic wind profile (23):

$u\left(z_{\mathrm{f}}\right)=a u\left(r_{\mathrm{ref}}\right)$.

From (23) with $z=z_{\mathrm{f}}$ and $z=z_{\text {ref }}$, we have

$$
\begin{gathered}
u\left(z_{\mathrm{f}}\right)=c \ln \frac{z_{\mathrm{f}}}{z_{0}}, \\
u\left(z_{\mathrm{ref}}\right)=c \ln \frac{z_{\mathrm{ref}}}{z_{0}},
\end{gathered}
$$

which gives the wind reduction factor $a$ from the roughness height $z_{0}$ and the interpolation height $z_{\mathrm{f}}$ as

$a=\frac{\ln \frac{z_{\mathrm{f}}}{z_{0}}}{\ln \frac{z_{\mathrm{ref}}}{z_{0}}}$.

The wind reduction factors for the 13 fuel categories are available in Baughman and Albini (1980, Table 3, p. 91). Equation (25) then allows to find the interpolation heights from the reduction factors as

$z_{\mathrm{f}}=\left(z_{\mathrm{ref}}\right)^{a}\left(z_{0}\right)^{1-a}$

In summary, we suggest two options for the vertical interpolation:

1. Interpolate to the fire mesh and then to the height $z_{\mathrm{f}}$ as described at the beginning of this section, and do not reduce the wind by the adjustment factor $a$. This option is preferable when $z_{1}<z_{\text {ref }}$, because otherwise some information is lost, and it may be used in any case.

2. Interpolate vertically to the common height $z_{\text {ref }}$, then horizontally to the fire mesh, and use the wind reduction factors $a$ given at the points of the fire mesh, following Eq. (24). This option is much simpler and cheaper, but it may lose information on the actual wind profile under the height $z_{\text {ref. }}$.

Both options are mathematically equivalent when the vertical resolution is so coarse that $z_{1} \geq z_{\text {ref }}$, as is usually the case except in microscale computations. Either option can be selected when running the code.

\subsection{Time step}

In each time step of the atmospheric model, the fire module is called from the third step (22) of the Runge-Kutta method in WRF-ARW. First, the wind is interpolated following Sect. 5.2 for use in the spread rate formula Eq. (1). The fire model then makes its own time step:

1. If there are any active ignitions, the level set function is updated and the ignition times of any newly ignited nodes are set following Sect. 3.6.

2. The numerical scheme (10-12) for the level set Eq. (9) is advanced to the next time step.

3. The time of ignition set for any any nodes that were ignited during the time step, from Eq. (13).

4. The fuel fraction is updated following Sect. 3.5.

5. The sensible and latent heat flux densities are computed from Eqs. (4) and (5) in each fire model cell. 
6. The resulting heat flux densities are averaged over the fire cells that make up one atmosphere model cell, and inserted into the atmospheric model, as described in the next Sect. 5.4.

The atmospheric model then completes the time step.

\subsection{Inserting fire heat fluxes into the atmosphere}

The heat fluxes from the fire are inserted into the atmospheric model as forcing terms in the differential equations of the atmospheric model into a layer above the surface, with assumed exponential decay with altitude. Such scheme is needed because WRF does not support flux boundary conditions. This is code originally due to Clark et al. (1996a,b) and it was rewritten for WRF variables in Patton and Coen (2004). The sensible heat flux is inserted as an additional source term to the equation for the potential temperature $\theta$, equal to the vertical divergence of the heat flux,

$\frac{d(\mu \theta)}{d t}(x, y, z)=R_{\Theta}(\Phi)+\frac{\mu(x, y) \phi_{h}(x, y)}{\sigma \varrho(x, y, z)} \frac{\partial}{\partial z} \exp \left(-\frac{z}{z_{\mathrm{ext}}}\right)$,

where $R_{\Theta}(\Phi)$ is the component of the source term (commonly called "tendency" in the WRF code) in the atmospheric model Eq. (21), $\sigma$ is the specific heat of the air, $\varrho(x, y, z)$ is the density, and $z_{\text {ext }}$ is the heat extinction depth, given as parameter fire_ext_grnd in namelist.input. The latent heat flux is inserted similarly into the source term of the vapor concentration $q_{\mathrm{m}}$ by

$$
\frac{d\left(\mu q_{\mathrm{m}}\right)}{d t}(x, y, z)=R_{Q_{\mathrm{m}}}(\Phi)+\frac{\mu(x, y) \phi_{q}(x, y)}{L \varrho(x, y, z)} \frac{\partial}{\partial z} \exp \left(-\frac{z}{z_{\mathrm{ext}}}\right),
$$

where $L$ is the specific latent heat of the air. Cf. Clark et al. (1996a, Eqs. 10, 12, 13, 18).

\section{Parallel structure and limitations}

Parallel computing is essential for fast execution, yet it imposes a significant constraint on user programming technique. At the danger of some simplification, one can say that WRF parallel infrastructure (Michalakes, 2000) essentially divides the domain horizontally into rectangular regions, called tiles, and different tiles are assigned to different processor cores, which execute in parallel. A numerical code in WRF needs to be tile callable. This means that the code runs on a single tile, using values from strips around the tile boundary in neighboring tiles, if necessary. The values in those strips may not be changed while the tile-callable code executes, and they are communicated by other code only after the tile-callable code exists. The communication in fact happens in two different ways, called MPI and OpenMP (Fig. 3), corresponding to distributed memory scheme (essentially, separate computers connected by a network) and shared memory (multiple processor cores in a single computer).

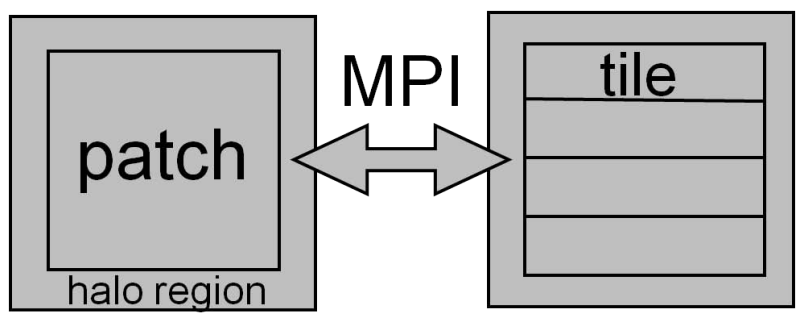

Fig. 3. Parallel communication in WRF. The computational domain is divided into disjoint rectangular patches. Each patch is updated by a single MPI process (distributed memory parallelism), and the process may read arary data in a strip around the patch, called halo region. The communication between the patches is by halo calls to the RSL parallel infrastructure (Michalakes, 2000), which update the halo regions by the values from the neighboring patches. Each patch may be divided into tiles, which execute in separate OpenMP threads (shared memory parallelism). Following WRF coding conventions (WRF Working Group 2, 2007), computational kernels execute in a single tile. They may read array values from a strip beyond the tile boundary but no explicit communication is allowed. 3-D arrays are divided into patches and tiles in the horizontal plane, cf., Fig. 1.

Consequently, numerical code must execute in stages, alternating between numerical computing and communicating values between the strips (Fig. 4), while going down the software layers (Fig. 5) in each stage to resume the computation. The fire module code executes in 6 stages interleaved with communication, 3 stages for initialization and 3 stages in every time step. For small tiles, the communication dominates the computational cost, which puts a practical limit on the number of processor cores that may be used for any given problem.

Unfortunately, the parallel computing structure limits the class of numerical methods that are feasible to implement. In particular, high-order methods, which need to update values at a node using values from distant nodes are no longer very practical, both because of the complexity of programming and because of the sharply increased communication cost. This explains our choice of numerical methods of the lowest possible order.

\section{Data input}

WRF and SFIRE may be run in both "ideal" and "real" modes, which require slightly different setups. Ideal runs were extended by adding fire-related capabilities, such as reading fuel, topography, and land use from simple text files. This allows to run simulations which go beyond what would normally be considered an ideal run and simplifies custom data input; the simulation of the FireFlux experiment (Sect. 9) was done in this way.

A WRF real run is used for forecasting and analysis of natural events. In real runs, the problem is set up from 


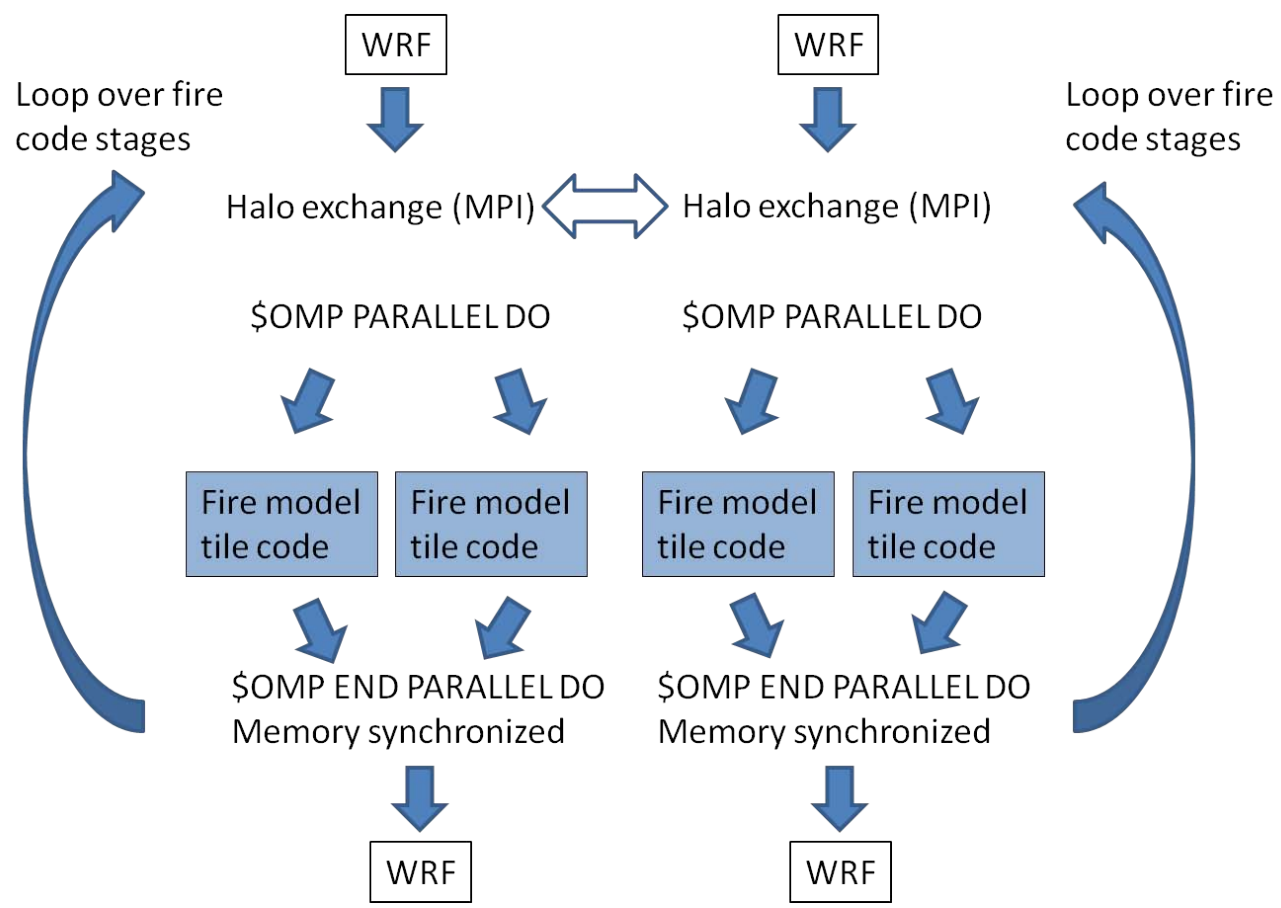

Fig. 4. Parallel structure of the fire module in the WRF physics layer. The core code itself executes on a single tile, with all communication done outside. Multiple passes through the fire module are needed in each time step.

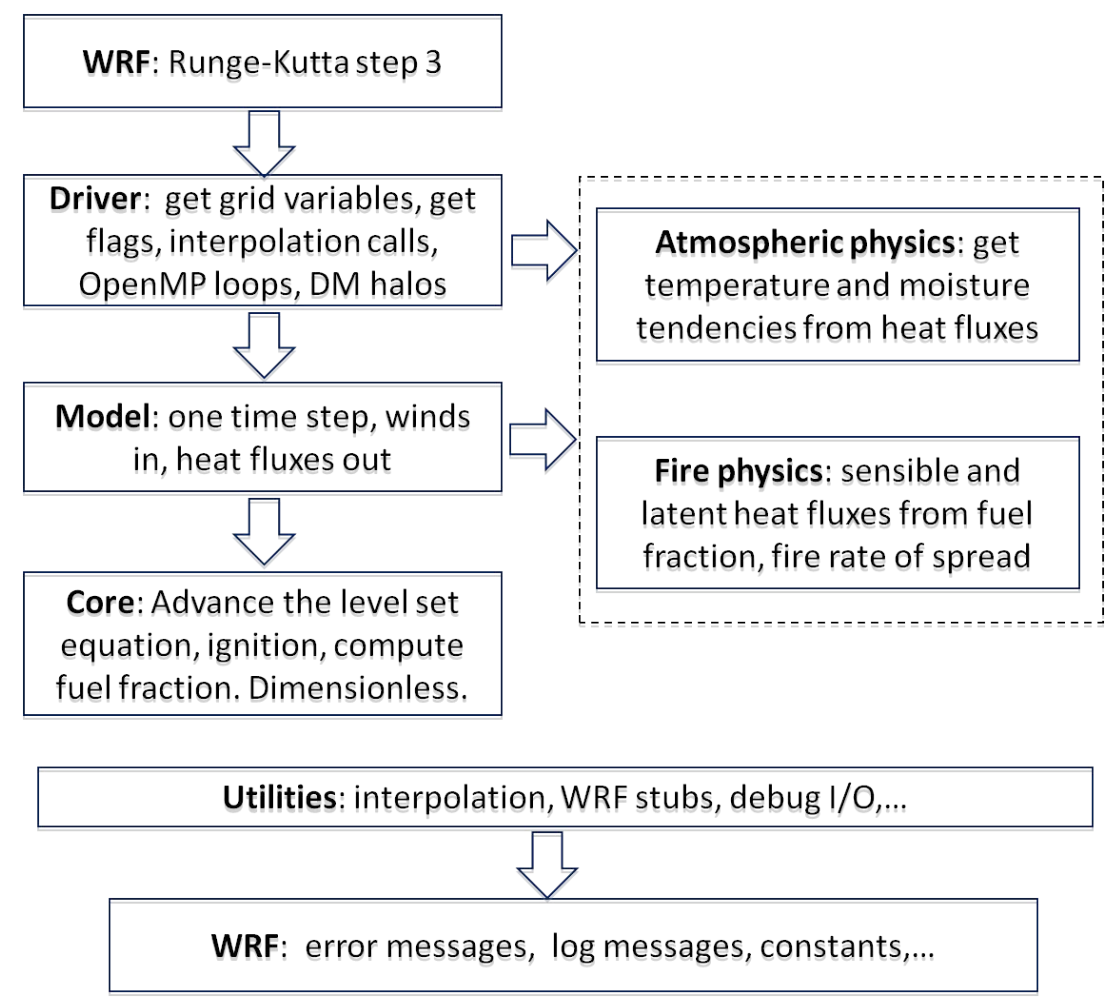

Fig. 5. Software layers of SFIRE. All physics dependencies are in the dashed box. The utilities layer is called from all the other layers above. 
the WRF Preprocessing System (WPS) (Wang et al., 2010, Chapter 3), which contains a number of utilities useful for preparing standard atmospheric and surface datasets for input into WRF. In a real run, a user must supply data for the initial and boundary conditions for the WRF simulation.

WPS has been extended (Beezley, 2011) with the ability to produce data defined on the refined surface meshes used by SFIRE (Sect. 8); however, it is not possible to distribute high resolution, global fields as is done in the standard dataset. Instead, the user must download any necessary high resolution fields and convert them for each simulation. The WPS extensions include the ability to input data in GeoTIFF format, which is a standard file format for high-resolution geoscientific data, such as aerial and satellite images. See http://www.openwfm.org/wiki/How_to_run_ WRF-Fire_with_real_data for details.

For a SFIRE simulation, it is only strictly necessary to download one additional dataset. This dataset contains the map of fuel categories on the simulation domain.. For simulations within the United States, this data can be obtained in GeoTIFF format from the USGS at http://www.landfire.gov. SFIRE uses an additional variable for high-resolution topography, which is allowed to be different from the topography used used by the atmospheric code. This is useful because a high resolution WRF simulation generally requires the topography to be highly smoothed in preprocessing for numerical stability. The fire code can benefit from a rougher topography for more accurate fire spread computations.

For atmospheric data, it is best to use the highest resolution dataset available to initialize a WRF simulation to capture as much of the local conditions near the fire as possible. Generally, publicly available atmospheric data is limited to around $10 \mathrm{~km}$ resolution. As a consequence, one should create several nested grids, each with a 3 to 1 refinement ratio, and a long spin-up prior to ignition in order to recreate local conditions. Preliminary results indicate that assimilation of data from weather stations or satellite radiances may be required for an accurate simulation (Beezley et al., 2010).

\section{WRF settings}

Since the coupled code initialization for the real cases does not differ from the one for the regular WRF, all physical and dynamical options available in the regular WRF are also available. Therefore, the same general rules apply to the configuration as to the configuration of the regular WRF.

\subsection{Domains and nesting}

However, one should keep in mind that resolutions of the finest domains in fire simulations are usually significantly higher than in weather forecasting applications. This has two consequences in terms of the proper WRF setup for SFIRE. First, if the resolution of any of the inner domains is less than
$100 \mathrm{~m}$, this domain should be actually resolved in the large eddy simulation (LES) mode, without the boundary layer parameterizations. At this resolution, the model should be able to resolve the most energetic eddies responsible for mixing within the boundary layer, so the boundary layer parameterization in this case is not needed. Second, since in the nested mode, vertical levels are common for all domains, the height of the first model level selected for the most outer (parent) domain, defines also the level of the first model layer for all inner (child) domains, even if their horizontal resolutions are an order of magnitude smaller. The fact that the vertical model resolution is the same for all domains significantly limits the minimum height above the ground of the first model level. This in turn is crucial for the fire model, which uses the wind speed interpolated to $6.1 \mathrm{~m}$ or less above the ground. Therefore, in the cases when the first model level must be relatively high above the ground it is recommended to perform only one way nesting, which allows the use of different vertical levels on different domains.

\subsection{Large Eddy Simulation and surface properties}

To enable the high-resolution simulation in Large Eddy Simulation (LES) mode, user should first disable the boundary layer parameterization (bl_pbl_physics=0). The LES mode requires the proper surface fluxes in order work properly. We recommend the option isf $f(x=1$, which makes WRF use a surface model to compute the surface fluxes. Other options with constant heat fluxes and drag are not well suited for fire simulations. Out of all surface exchange parameterizations only the classic Monin-Obukhov theory (sf_sfclay_physics=1) is recommended for the LES cases. This option assures a proper computation of surface transfer coefficients that are used together with the surface properties (provided by the surface model) for computation of the surface fluxes of the momentum, heat and moisture. The surface model itself computes properties of the surface, but does not compute the surface exchange coefficients, which are needed for computation of the surface fluxes. Hence, in order to compute them, the surface properties must be provided by a surface model, which is enabled by choosing a non-zero sf_surface_physics. The subgrid scale parameterization used by the WRF in LES mode is defined by the km_opt parameter, which should be set to 2 (TKE closure), or 1 (Smagorinsky scheme).

In real cases, proper initialization for the selected land surface model is provided automatically, and all other components. In ideal cases, users are responsible for the proper initialization and setting land use properties.

\subsection{Mesh resolution and refinement ratios}

The fire mesh needs to be about 10 times finer than the atmospheric mesh to allow for gradual heat release into the atmosphere, even if fuel and topography data may not be available 

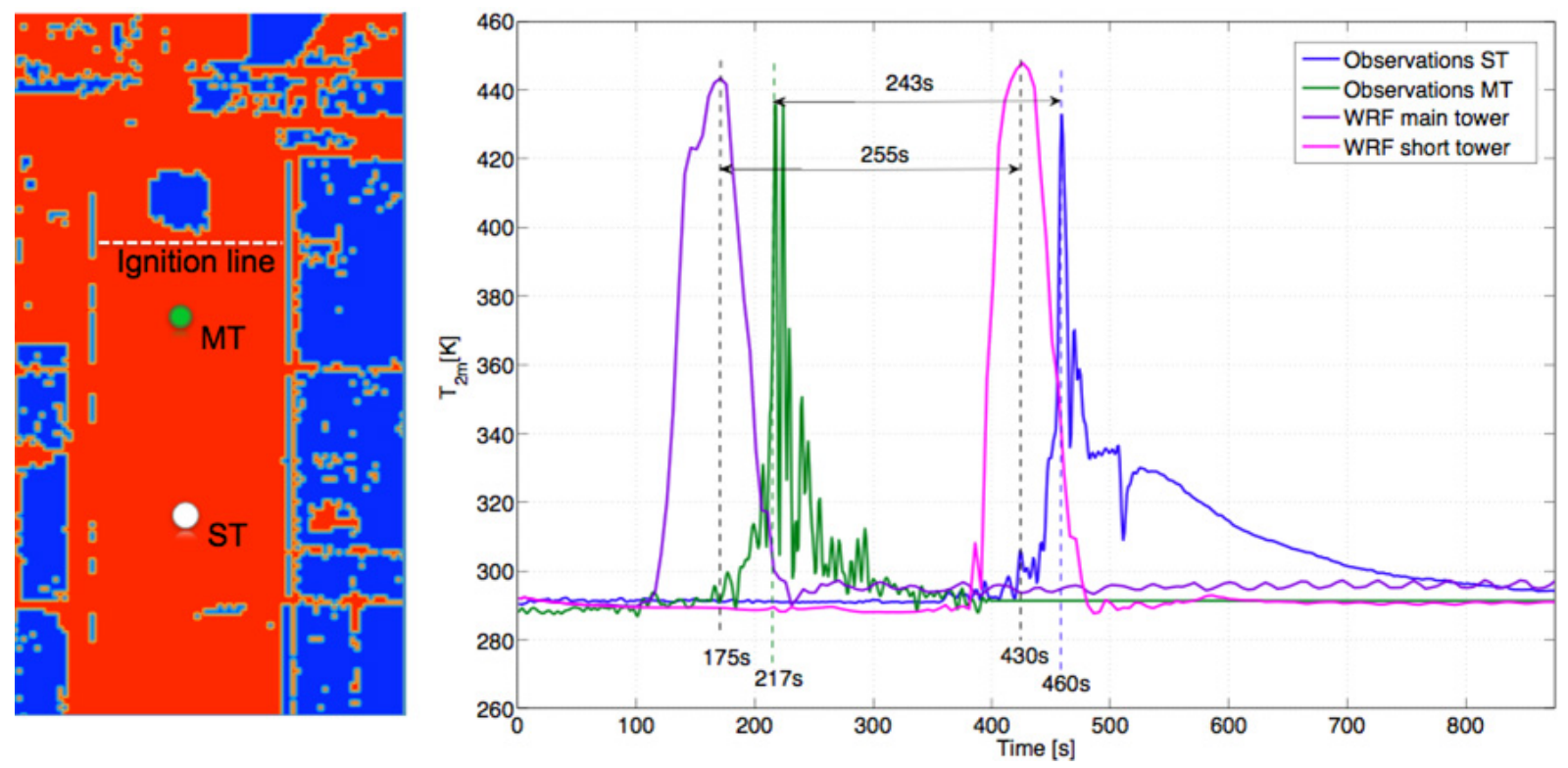

Fig. 6. Simulation of the FireFlux experiment (Clements et al., 2007) by WRF and SFIRE. Left: map of landuse category for the experimental plot, with the ignition line and the observation towers marked. Right: simulated and measured temperature profiles at the location of the observation towers. The simulated fire propagation takes $243 \mathrm{~s}$ from tower MT to tower ST, while the measured time is $255 \mathrm{~s}$ ( $4.7 \%$ difference). Due to the instrument failure that took place during the experiment about $20 \mathrm{~s}$ after the fire front passage, the recorded temperature drop is unrealistically slow, and do not represent the actual cooling phase. Therefore, the discrepancies in the temperature drop at the short tower should not be treated as an indication of the model error. The turbulent kinetic energy and the wind profile at that tower were captured well and they do not indicate specific problems associated with realistic rendering of the post fire cooling. From Kochanski et al. (2010).

at such fine resolution. The atmospheric mesh step should be about $60 \mathrm{~m}$ or less for proper feedback of the wind on the fire line. Larger mesh step was reported to result in too large fire spread rates in interaction with the atmosphere (Clark et al., 1996a, p. 887); however, we did not observe this.

Because of the computational cost, the practical limit of the atmospheric domain resolution for forecasting applications seems to be currently around $400 \mathrm{~m}$. At that resolution, the simulation to wall clock time ratio may be kept around 6 , that is, $24 \mathrm{~h}$ forecast may be completed within $4 \mathrm{~h}$. The limiting factor is the short time step required for higher resolutions. Using more processor cores (Sect. 6) for a fixed problem eventually reaches the point of diminishing return, because each core gets a smaller domain to work on and the parallel efficiency decreases. One should keep in mind that even this relatively coarse atmospheric resolution (from the fire modeling point of view) already extends beyond the maximum resolution of the standard meteorological static surface data, which is currently $1 \mathrm{~km}$. At the atmospheric model resolution of $400 \mathrm{~m}$, the refinement ratio of 10 brings the fire model mesh size close to maximum resolution of the available fuel data, which is currently $30 \mathrm{~m}$. So, from the fuel data point of view, running real forecasting simulations at higher than $30 \mathrm{~m}$ resolutions does not seem practical. However, since the fire spread depends on the slope gradient that is computed on the fire mesh, using finer fire model mesh in complex terrain may be justified. The topographical data are generally available at higher resolutions than the fuel maps (for the US, it is $2 \mathrm{~m}$ ), so in cases when the sub-grid variability in the fuel composition is expected to be relatively small, and the topographical effects are expected to be important, further increasing of the fuel model resolution beyond the $30 \mathrm{~m}$ limit may be desirable.

From the point of view of the atmosphere-fire interaction, a coarser horizontal and vertical atmospheric resolution means less intense feedback from fires of the burning area smaller than the atmospheric grid cells, since the fire heat flux computed on the fire mesh gets averaged over a bigger atmospheric cell. Smaller heat flux leads to weaker fire-induced updraft, less intense surface convergence and finally weaker wind speed up at the fire front, which in turn could theoretically result in underestimation of the fire rate of spread. However, since the Rothermel fire model was calibrated, based on undisturbed wind speed measured upwind from the fireline at $6.1 \mathrm{~m}$ height, the local speed up at the fire line has been already captured by the model constants. Whether further adjustments to the fire parameterization are needed, depending on the resolution and fire-atmosphere refinement ratio, is a question for future research.

For real simulations, where the errors in fuel description are much more severe, the resolution issue does not have to be critical. However, in fine-resolution simulations focused 

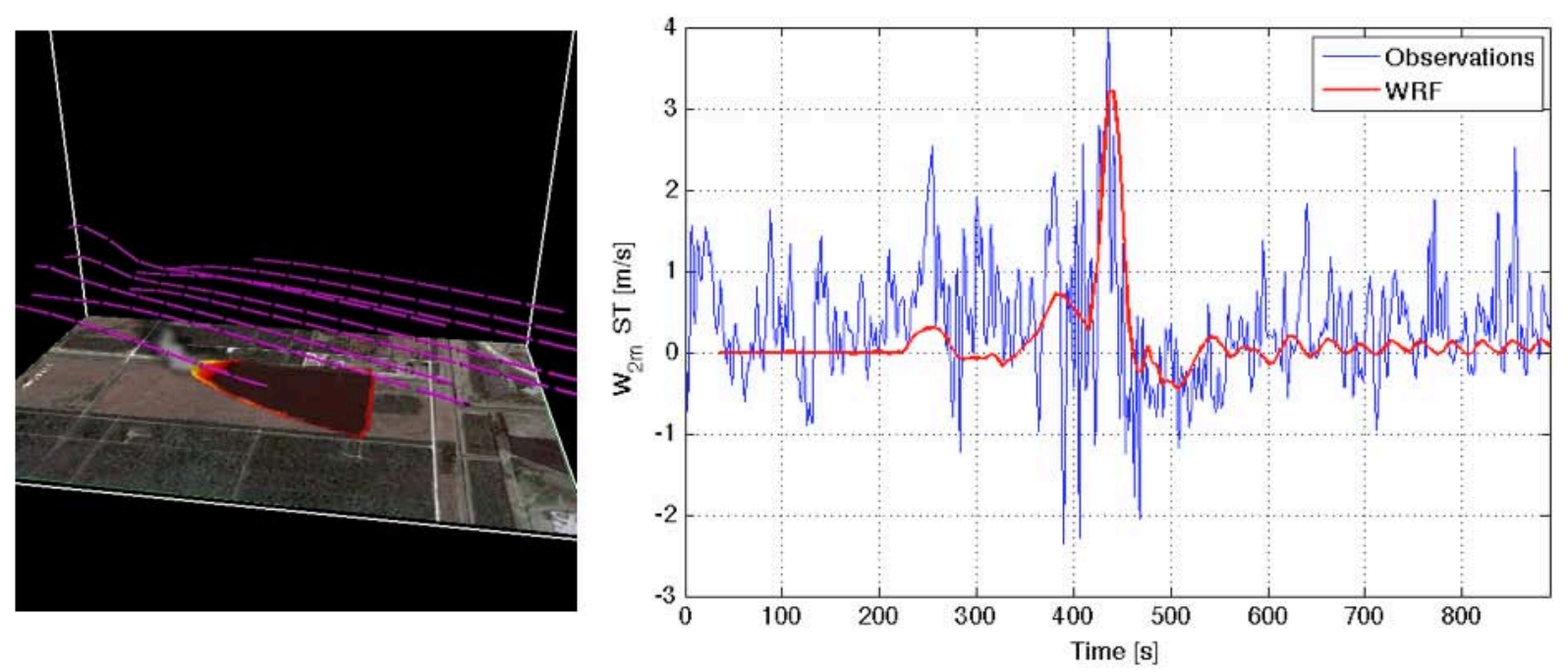

Fig. 7. Simulation of the FireFlux experiment (Clements et al., 2007) by WRF and SFIRE. Left: surface heat flux and selected flowlines. Visualization in VAPOR by Bedřich Sousedík. Surface image from Google Earth. Right: vertical velocity at $2 \mathrm{~m}$ height at tower ST. (See Fig. 6 left for location.) The simulation shows a good agreement with the experiment. From Kochanski et al. (2010).

on the atmosphere-fire coupling with well-known fuel conditions, such as the FireFlux experiment (Figs. 6 and 7 ), realistic rendering of the atmospheric response to the surface heating associated with fire is absolutely crucial. For these applications, the fire model should use the wind speed taken from the level as close to the mid-flame height as possible. This requirement translates into a need for very high vertical resolution. For realistic modeling of the fire of expected flame height of let us say $4 \mathrm{~m}$, the first atmospheric model level should be at $2 \mathrm{~m}$. Updrafts associated with fire plumes may easily reach a rising speed of $5 \mathrm{~m} \mathrm{~s}^{-1}$, so in order to keep the simulation numerically stable, the time step should not be greater than $0.4 \mathrm{~s}$. Assuming the horizontal wind speeds below $25 \mathrm{~m} \mathrm{~s}^{-1}$, the vertical model resolution will be a limiting factor in terms of the time step as long as the horizontal grid spacing will be greater than $10 \mathrm{~m}$. This reasoning was applied during the design of the FireFlux simulation, for which the horizontal resolution has been set to $10 \mathrm{~m}$, as an optimal value providing high horizontal resolution yet not requiring further reduction in the time step. Detailed analysis of the FireFlux experiment is in progress and will be published as a separate case study elsewhere.

Since the fine-resolution simulations are mostly run in the LES mode as opposed to the coarser real cases that rely on boundary layer parameterization, the degree to which the vertical mixing is captured by the model directly depends on the model resolution. From that point of view, the grid refinement for fine-resolution cases should be expected to bring more benefits than for real cases utilizing boundary layer and cloud parameterizations, which were originally designed for much coarser atmospheric meshes.

\subsection{Time step}

In real SFIRE simulations performed in multi-domain configurations the time step requirements for the outer domains (run without fire) do not differ from general meteorological cases. The recommended time step of 6 times the horizontal grid spacing (in $\mathrm{km}$ ) may be used as a starting point. However, for the finest domains run with fire simulations, the time step in most cases must be significantly smaller. For domains with low vertical resolution and simple topography, the horizontal mesh step is crucial for numerical stability, since the horizontal velocity is greater than the vertical one. In fire simulations with high vertical resolution, the vertical velocity induced by fire may violate the CFL condition. Therefore, it is advisable to use a vertically stretched grid, with finer resolution at the surface (where updraft velocities are not that high) and lower resolution at higher levels where stronger updrafts are expected. This allows for having the first model level relatively close to the ground, yet with vertical spacing aloft big enough to handle strong convective updrafts without violating the CFL condition.

In real cases, the pressure levels may be defined directly. In ideal SFIRE runs, there is now an option which turns on hyperbolic grid stretching. One should keep in mind that running the SFIRE simulations with high-resolution topography in most cases limits the maximum numerically stable time step. Steep terrain often induces high vertical velocities that may violate the CFL condition. Therefore, these cases usually require significantly smaller time steps than similar simulations run with low-resolution, smooth topography. 


\section{Computational simulations}

\subsection{Examples of output and computational results}

Kim (2011) has verified that the level set method in the fire module advects the fire shape correctly, on some of the same examples that were used to verify the tracer code in CAWFE (Clark et al., 2004).

A number of successful simulations with SFIRE now exist. Jenkins et al. (2010) have demonstrated fireline fingering behavior for a sufficiently long fireline (Figs. 8 and 9) on an ideal example, with similar results as in Clark et al. (1996a,b). Kochanski et al. (2010) have demonstrated the validity of SFIRE on a simulation of the Clements et al. (2007) FireFlux grass fire experiment and obtained good agreement with data (Figs. 6 and 7). Dobrinkova et al. (2011) simulated a fire in Bulgarian mountains using real meteorological and geographical data, and ideal fuel data. Beezley et al. (2010) simulated the 2010 Meadow Creek fire in Colorado mountains using real data from online sources. Topography (Fig. 10) at up to $3 \mathrm{~m}$ horizontal resolution was obtained from the National Elevation Dataset (NED, http://ned.usgs.gov) and fire fuel datasets from Landfire (http://landfire.cr.usgs. gov) at up to $10 \mathrm{~m}$ resolution. Six nested domains were required to scale the simulation down from the atmospheric initialization $(32 \mathrm{~km})$ to the fire grid resolution $(10 \mathrm{~m})$. Cloud physics was enabled in domains $1-3$. The fire mesh refinement ratio was 10 times on the finest domain to capture fire surface variables and for a gradual release of the heat flux near the fireline. Realistic fire and atmosphere behavior was obtained (Figs. 11 and 12).

\subsection{Validation plan}

Validation of a coupled atmosphere-fire model used for simulation of wild fires is very challenging. The biggest problem arises from the fact that the amount of measurement data that can be used for model validation is very limited. The laboratory-scale fire experiments performed in wind tunnels cannot be used directly as a benchmark for a model simulating wildland fires, since in the laboratory-scale fires tend to behave differently than in the open atmosphere (Beer, 1991; Mell et al., 2007). Therefore, for the validation of the coupled model, we rather plan to use data collected during field experiments, and start from relatively simple cases, where there are not as many conflicting influences. We also foresee that the validation process will uncover the need to add further parametrizations of certain fire behaviors which are not currently captured.

The basic evaluation of the the model capability to simulate realistically the fire front shape and its propagation through a uniform fuel bed could be performed based on the data collected during the Australian Grass Fire Experiment. However, we realize that the limitations of this data set would not allow for full investigation of the model capabilities and

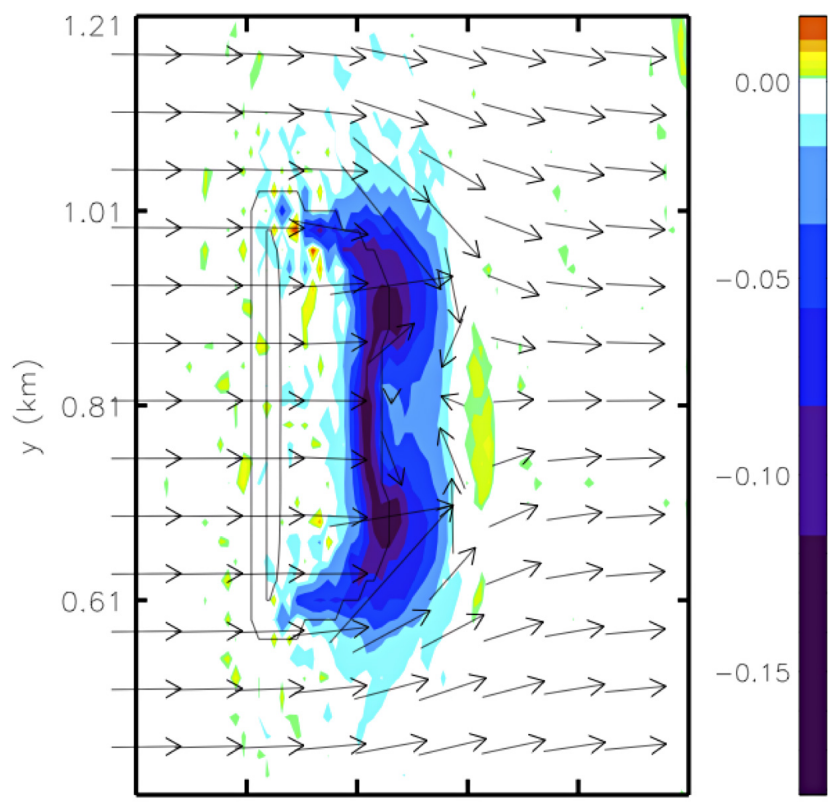

Fig. 8. $\mathrm{X}-\mathrm{Y}$ section of wind vector at $18 \mathrm{~m}$ and pressure perturbation $240 \mathrm{~s}$ after line ignition, initialized with uniform wind profile. The fire develops two fingers due to wind direction inversion in the middle. From Jenkins et al. (2010).

deficiencies. During this experiment, the wind measurements were taken only upwind from the actual fire, and only at two levels. Therefore, they do not provide a full description of the vertical wind profile which may affect the fire rate of spread through the atmosphere-fire coupling (Jenkins et al., 2010), and they lack the information about the actual wind at the fire line. Since the coupled fire-atmosphere model computes the fire rate of spread based on the local wind, it is absolutely crucial for its validation to know not only the fire spread rate but also the local wind speed.

Therefore, for the first model validation, we plan to use the data collected during the FireFlux experiment (Clements et al., 2007). Preliminary results (Kochanski et al., 2010) show that WRF coupled with SFIRE is capable of realistic rendering of the rate of the fire spread, as well as temperature, upward velocities, and horizontal wind speed associated with a steady fire front passage (not affected directly by the ignition). Some early results are included here (Figs. 6 and 7). Unfortunately, the lack of full infrared documentation of the fire front evolution does not allow for an evaluation of the model in terms of a realistic representation of the fire front shape. We also consider using data collected during the Meteotron experiment (Benech, 1976) to validate explicitly the simulated plume dynamics. However, it seems that the FireFlux is more appropriate since it provides data collected during the passage of the real fire, while Meteotron experiment focused on the dynamics of a stationary plume generated by a set of burners. 


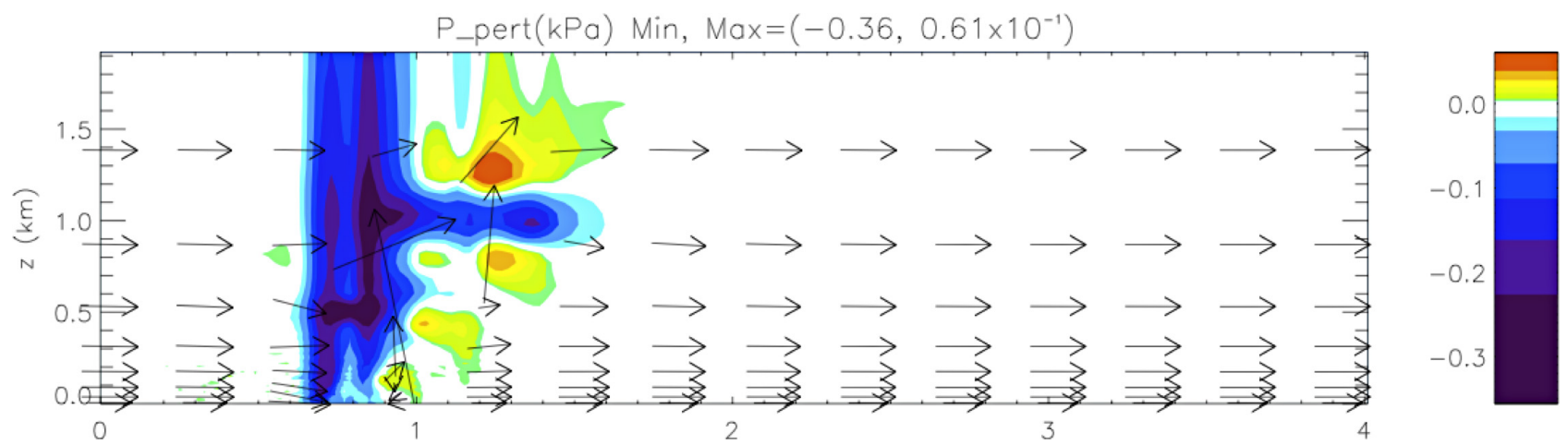

Fig. 9. X-Z section of wind vector and pressure perturbation at the centerline for the fire in Fig. 8. From Jenkins et al. (2010).
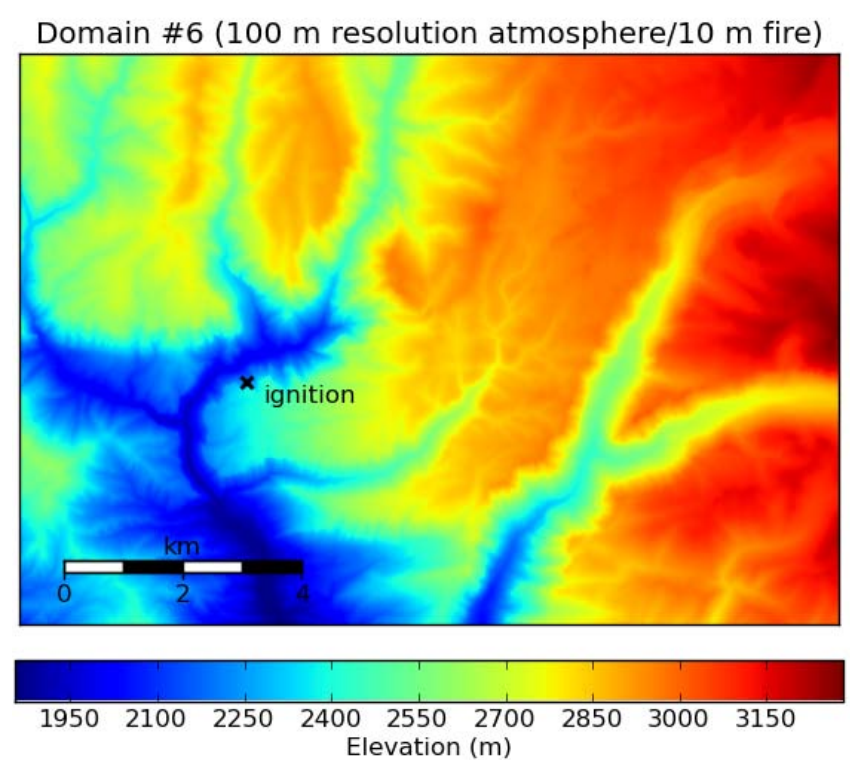

Fig. 10. Topography of the finest domain of the Meadow Creek fire simulation. From Beezley et al. (2010).

Another validation venue is the use of data from targeted experiments, arranged with our collaborators. Such experiments could be directed to answer specific questions about fire behavior, which SFIRE is not able to model at the moment, e.g., how does ignition from a small fire in grass behave before the fire reaches the full wind-driven spread rate, predicted by Rothermel's formula.

Finally, we plan to perform the overall evaluation of the model in real fire cases. We are currently working on the validation based on the observed fire perimeters during Harmanli fire (Bulgaria), Meadow Creek Fire (Colorado), and Witch fire (California). Unfortunately, in most real fire cases, no meteorological data directly at the fire are available, so the only available information that may be used for model evaluation is the final fire perimeter, and, in some cases, a progression of recorded perimeters.

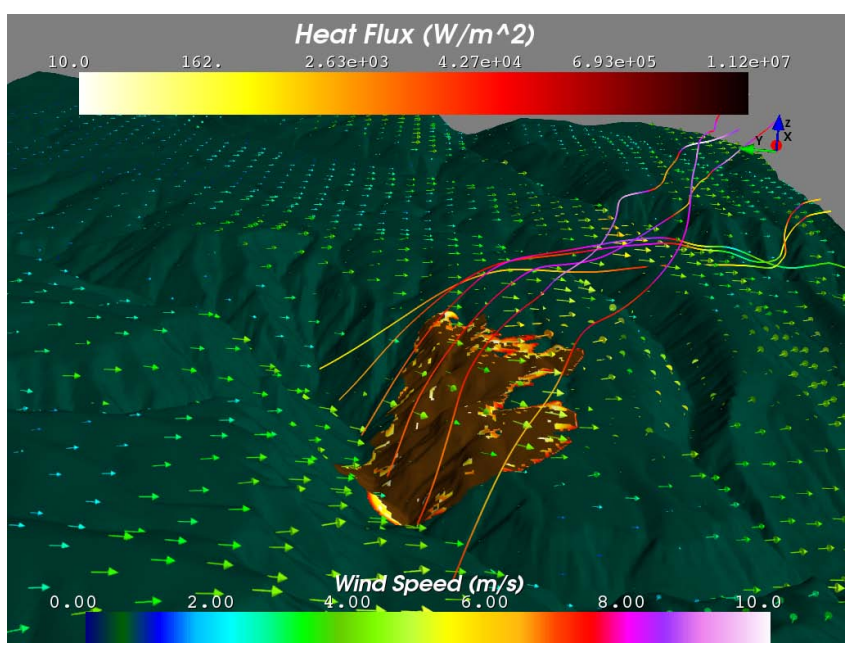

Fig. 11. The finest domain in the Meadow Creek fire simulation $5 \mathrm{~h}$ after ignition. Unburned fuel is displayed as green, burned fuel as brown. The heat flux from the fire appears near the fire line. Arrows indicate the surface winds, while streamlines show the atmospheric winds flowing over the fire region. Visualization in MayaVi. From Beezley et al. (2010).

\section{Conclusions}

We have described the atmosphere-fire model consisting of WRF coupled with SFIRE. The software is publicly available and it supports both ideal and real runs. Visualization and diagnostic utilities are available. Currently, the model is suitable for research and education purposes. Validation is in progress.

\subsection{Additional features}

SFIRE does not yet support canopy fire, although canopy fire colocated with ground fire is contained in CAWFE. The reason was the desire to keep the code as simple as possible early on and add features only as they can be verified and validated. The support for canopy fire will be added in 


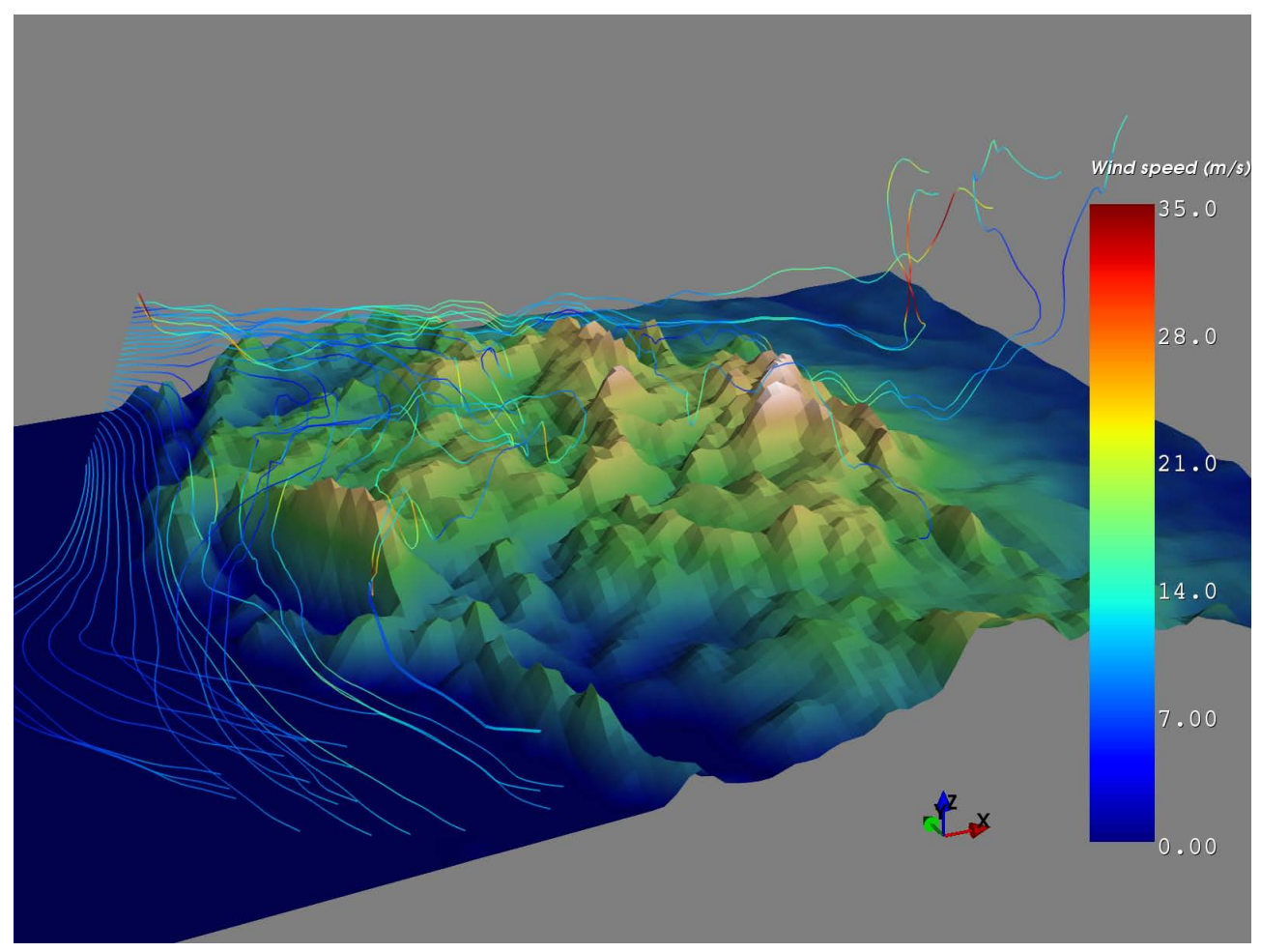

Fig. 12. The top level domain in the Meadow Creek fire simulation $5 \mathrm{~h}$ after ignition. Streamlines show the winds blowing East, over the Rocky Mountains and South down the coast of California. Visualization in MayaVi. From Beezley et al. (2010).

future. Adding smoke from the fire to WRF is also under consideration. There are two possible approaches to implementation of the smoke dispersion in WRF and SFIRE. The basic one would be to treat the smoke as a passive tracer advected by the wind, while the more advanced (taking into account its chemical reactivity during its transport), would require a coupling between the SFIRE and WRF-Chem. A list of desired features and a record of the progress of the development are maintained at http://www.openwfm.org/wiki/ OpenWFM_development_notes.

\subsection{Atmosphere}

Rothermel's spread model (Eq. 1) assumes wind as if the fire was not there. In practice, the wind was measured away from the fire. In a coupled model, however, the feedback on the fire is from the wind that is influenced by the fire. Clark et al. (2004) noted that the horizontal wind right above the fireline may even be zero, and proposed to take the wind from a specified distance behind the fireline. Also, the strong heat flux from fire disturbs the logarithmic wind profile, and the rate of spread as a function of wind at a specific altitude may not be a good approximation; rather, the fire spread may depend more strongly on the complete wind profile (Jenkins et al., 2010) and on turbulence (Sun et al., 2009). The assumption of horizontal homogeneity in the Monin-Obukhov similarity theory is not satisfied here; the horizontal dimension of the active part of fire is not orders of magnitude larger than the boundary layer height as required, and it may be in fact smaller. Another indication that the Monin-Obukhov theory may not apply for fires is a strong drop in the heat transfer in the case of strong temperature gradients, shown in our early tests.

Horizontal wind could be interpolated vertically to different heights for different fuels like in CAWFE model, which takes the wind from different mesh levels for different fuels. However, here we follow a classical approach of Rothermel (1972) and Baughman and Albini (1980), where the wind speed is evaluated at the common $6.1 \mathrm{~m}$ height, and then converted to the mid-flame height using the fuel-specific wind correction factors.

Very strong vertical components of the wind caused by the fire result in the need for short time steps to avoid violation of the vertical CFL condition (Sect. 8.4). It would be interesting to couple the fire module also with the Non-hydrostatic Mesoscale Model (NMM) core of WRF, which is implicit in the vertical direction (Janjic et al., 2005), and it may perform better in the presence of strong convection (Litta and Mohanty, 2008). The ARW dynamical core (Skamarock et al., 2008), which we currently use, is semi-implicit in the vertical direction in the vertical wind component and the geopotential. 


\subsection{Fire}

The more recent Scott and Burgan (2005) fuel categories are more detailed than Anderson (1982) categories, they are supported by BehavePlus, and fuel maps using them are available from Landfire. But instead of describing additional categories in namelist. fire, it may be more useful to support the import of fuel files from BehavePlus, which is also well suited for editing and diagnosing fuel models. More accurate fuel models (Albini et al., 1995; Clark et al., 1996a), including those in BehavePlus, consider fuels to be mixtures of components with different burn times, which results in a different heat release curve.

While the spread rate of established fire in the simulation of the FireFlux experiment was reasonably close, the simulated fire still arrived at the observation towers too soon (Kochanski et al., 2010), because it started too quickly. A better parametrization of the ignition process seems to be in order. The fire spread in the Meadow Creek fire simulation was also too fast, but for a different reason. It is well known that the actual spread rates of wildland fires tend to be lower than the spread rates in simulations, which are derived from laboratory experiments. This effect might be attributed to irregularities on scales not captured by the simulation (Finney, 1998 , p. 34), including granularity of the fuel supply not reflected in the data. Refining the semi-empirical model from detailed numerical simulations and parametrizing complex fire behavior are suggested important research areas.

The computation of the heat fluxes in Eqs. (4) and (5) does not take into account the evaporation of moisture present in the fuel, only the production of water by burning of hydrocarbons. This error is typically just few \%, however, which is small in comparison with other uncertainties. As the fuel moisture content can be significant in some ecosystems, it will be treated explicitly in a future version of the code. The fuel models should be dynamic (with variable fuel moisture) as in BehavePlus. Coen (2005) added an explicit diurnal cycle for the moisture into CAWFE. Here, moisture content could be coupled with existing WRF land surface models, which could take into account air humidity and precipitation. The radiative and convective parts of the sensible heat flux should be treated differently. The release of surface heat and moisture into the atmosphere are already present in WRF soil models. Their scale, however, is different from the powerful heat release from a fire.

Spotting (secondary ignitions by wind-lofted firebrands) could be modeled as additional point igntions, created dynamically. Deciding when and where the secondary ignitions occur, however, would require stochastic approaches, possibly modeling the spotting location and frequency as a random variable dependent on the fire location, distance, and the wind, similarly as in Mandel et al. (2004a).

\subsection{Numerical methods}

In a numerical implementation, the level set method is global, unlike tracers, which move locally. In spite of the fact that the level set equation determines the fire spread locally from the spread rate at the fireline, the behavior of the fireline depends slightly on the wind, the fuel, and the level set function in certain other locations from previous time steps, because of the discretization errors and the artificial diffusion. This nonlocal behavior has not been practically significant, however.

The fuel fraction calculation (18) can have significant error in the fire mesh cells near the fireline, which will to some degree average out over the atmospheric mesh cells. Rigorous error analysis will be done elsewhere. We are currently testing an alternative method which is always first order in the sense that it is exact when the time from ignition and the level set function are linear in space. The alternative method is more computationally expensive, but, on the other hand, it might allow to decrease the fire mesh refinement ratio; with large meshes, it is possible to run against 32 bit integer limits.

\subsection{Data assimilation}

Data assimilation for wildland fires is an area of great interest. Methodologies for a reaction-diffusion model were proposed based on the ensemble Kalman filter (EnKF) and the particle filter (Mandel et al., 2004b). Unfortunately, statistical perturbations can cause spurious fires, which do not dissipate. Combination of the EnKF with Tikhonov regularization alleviates the problem somewhat (Johns and Mandel, 2008; Mandel et al., 2009), but the resulting method is still not robust enough. A new method, called morphing EnKF and based on combined amplitude and displacement correction (Beezley and Mandel, 2008), was shown to work with WRF and SFIRE (Mandel et al., 2009), and it is under continued development (Mandel et al., 2010, 2011). We are not aware of any work elsewhere on data assimilation for a coupled fire-atmosphere model. Particle filters were proposed for discrete cell-based fire models (Bianchini et al., 2006; $\mathrm{Gu}$ et al., 2009), using fitness functions involving the area burned rather than intensities of physical variables.

Starting the model from a known fire perimeter is important for many potential users. This can be understood as a data assimilation problem, but we are considering a simpler method for this particular case: prescribe the fire history up to the time of the given perimeter to allow the atmospheric conditions to evolve, then allow the coupled model take over. Tools to produce such artificial fire history are being developed (Kondratenko et al., 2011). Possibly the simplest alternative is an interpolation of the ignition time between a given ignition point and the perimeter. A more complex version would run the fire model (without atmosphere) backwards in time and attempt to find the ignition point automatically. The latter approach could be also interesting for forensic purposes. 


\section{Supplementary material related to this article is available online at: http://www.geosci-model-dev.net/4/591/2011/ gmd-4-591-2011-supplement.zip.}

Acknowledgements. The authors would like to thank John Michalakes for developing the support for the refined surface fire grid in WRF and information about WRF algorithms, Ned Patton for providing a copy of his prototype code, Janice Coen for providing a copy of CAWFE, liason with NCAR, and useful suggestions, and Volodymyr Y. Kondratenko for implementing the interpolation of ignition time in Sect. 3.5, as well as other assistance. Other contributions to the model are acknowledged by bibliographic citations in the text. We would like to thank also Mary Ann Jenkins for reading this paper and suggesting improvements. This research was supported by NSF grant AGS-0835579 and NIST Fire Research Grants Program grant 60NANB7D6144.

Edited by: D. Lawrence

\section{References}

Albini, F. A. and Reinhardt, E. D.: Modeling ignition and burning rate of large woody natural fuels, Int. J. Wildland Fire, 5, 81-91, doi:10.1071/WF9950081, 1995.

Albini, F. A., Brown, J. K., Reinhardt, E. D., and Ottmar, R. D.: Calibration of a Large Fuel Burnout Model, Int. J. Wildland Fire, 5, 173-192, doi:10.1071/WF9950173, 1995.

Anderson, H. E.: Aids to determining fuel models for estimating fire behavior, General Technical Report INT-122, US Department of Agriculture, Forest Service, Intermountain Forest and Range Experiment Station, available at: http://www.fs.fed.us/rm/pubs_int/ int_gtr122.html (last access: 4 July 2011), 1982.

Andrews, P. L.: BehavePlus fire modeling system: past, present, and future, Paper J2.1, 7th Symposium on Fire and Forest Meteorology, available at: http://ams.confex.com/ams/pdfpapers/ 126669.pdf (last access: 4 July 2011), 2007.

Baughman, R. G. and Albini, F. A.: Estimating Midflame Windspeeds, in: Proceedings, Sixth Conference on Fire and Forest Meteorology, Seattle, WA April 22-24, 1980, Society of Americal Foresters, Washington, DC, 88-92, 1980.

Beer, T.: The interaction of wind and fire, Bound.-Lay. Meteorol., 54, 287-308, 1991.

Beezley, J. D.: Importing High-resolution Datasets into Geogrid, Paper P2, 12th WRF Users' Workshop, National Center for Atmospheric Research, June 20-24, 2011, 2011.

Beezley, J. D. and Mandel, J.: Morphing Ensemble Kalman Filters, Tellus, 60A, 131-140, doi:10.1111/j.1600-0870.2007.00275.x, 2008.

Beezley, J. D., Kochanski, A., Kondratenko, V. Y., Mandel, J., and Sousedík, B.: Simulation of the Meadow Creek fire using WRFFire, Poster at AGU Fall Meeting 2010, available at: http://www. openwfm.org/wiki/File:Agu10_jb.pdf (last access: 4 July 2011), 2010.

Benech, B.: Experimental Study of an Artificial Convective Plume Initiated from the Ground, J. Appl. Meteorol., 15, 127-137,
doi:10.1175/1520-0450(1976)015<0127:ESOAAC > 2.0.CO;2, 1976.

Bianchini, G., Cortés, A., Margalef, T., and Luque, E.: Improved Prediction Methods for Wildfires Using High Performance Computing: A Comparison, in: Computational Science, ICCS 2006, edited by: Alexandrov, V., van Albada, G., Sloot, P., and Dongarra, J., Vol. 3991 of Lecture Notes in Computer Science, Springer Berlin / Heidelberg, 539-546, doi:10.1007/1175850173, 2006.

Clark, T. L., Jenkins, M. A., Coen, J., and Packham, D.: A Coupled Atmospheric-Fire Model: Convective Feedback on Fire Line Dynamics, J. Appl. Meteorol., 35, 875-901, doi:10.1175/15200450(1996)035<0875:ACAMCF>2.0.CO;2, 1996a.

Clark, T. L., Jenkins, M. A., Coen, J. L., and Packham, D. R.: A coupled atmosphere-fire model: Role of the convective Froude number and dynamic fingering at the fireline, Int. J. Wildland Fire, 6, 177-190, doi:10.1071/WF9960177, 1996b.

Clark, T. L., Coen, J., and Latham, D.: Description of a Coupled Atmosphere-Fire Model, Int. J. Wildland Fire, 13, 49-64, doi:10.1071/WF03043, 2004.

Clements, C. B., Zhong, S., Goodrick, S., Li, J., Potter, B. E., Bian, X., Heilman, W. E., Charney, J. J., Perna, R., Jang, M., Lee, D., Patel, M., Street, S., and Aumann, G.: Observing the dynamics of wildland grass fires - FireFlux - A field validation experiment, Bull. Am. Meteorol. Soc., 88, 1369-1382, doi:10.1175/BAMS88-9-1369, 2007.

Coen, J. L.: Simulation of the Big Elk Fire using coupled atmosphere-fire modeling, Int. J. Wildland Fire, 14, 49-59, doi:10.1071/WF04047, 2005.

Coen, J. L. and Patton, N.: Implementation of Wildland Fire Model Component in the Weather Research and Forecasting (WRF) Model, available at: http://www.mmm.ucar.edu/research/ wildfire/wrf/wrf_summary.html (last access: December 2010), 2010.

Dobrinkova, N., Jordanov, G., and Mandel, J.: WRF-Fire Applied in Bulgaria, in: Numerical Methods and Applications, edited by Dimov, I., Dimova, S., and Kolkovska, N., vol. 6046 of Lecture Notes in Computer Science, Springer, Berlin/Heidelberg, 133140, doi:10.1007/978-3-642-18466-615, 2011.

Dudhia, J.: The Weather Research and Forecasting Model: 2010 Annual Update, 2010 WRF Users Workshop, available at: http://www.mmm.ucar.edu/wrf/users/workshops/WS2010/ abstracts/1-1.pdf (last access: 4 July 2011), 2010.

Filippi, J. B., Bosseur, F., Mari, C., Lac, C., Moigne, P. L., Cuenot, B., Veynante, D., Cariolle, D., and Balbi, J.-H.: Coupled atmosphere-wildland fire modelling, J. Adv. Model. Earth Syst., 1, Art. 11, doi:10.3894/JAMES.2009.1.11, 2009.

Finney, M. A.: FARSITE: Fire Area Simulator-model development and evaluation, Res. Pap. RMRS-RP-4, Ogden, UT: U.S. Department of Agriculture, Forest Service, Rocky Mountain Research Station, available at: http://www.fs.fed.us/rm/pubs/rmrs_rp004. html (last access: 4 July 2011), 1998.

$\mathrm{Gu}, \mathrm{F}$., Yan, X., and Hu, X.: State estimation using particle filters in wildfire spread simulation, in: SpringSim '09: Proceedings of the 2009 Spring Simulation Multiconference, Society for Computer Simulation International, San Diego, CA, USA, 1-8, 2009.

Janjic, Z., Black, T., Pyle, M., Chuang, H.-Y., , Rogers, E., and DiMego, G.: The NCEP WRF NMM Core, 5th WRF/14th MM5 User's Workshop, paper 2.9, available 
at: http://www.mmm.ucar.edu/wrf/users/workshops/WS2005/ abstracts/Session2/9-Janjic.pdf (last access: 4 July 2011), 2005.

Jenkins, M., Kochanski, A., Krueger, S. K., Mell, W., and McDermott, R.: The Fluid Dynamical Forces Involved in Grass Fire Propagation, Poster at AGU Fall Meeting 2010, available at: http://www.openwfm.org/wiki/File:AGU.2010.poster. jenkins.etal.pdf (last access: 4 July 2011), 2010.

Johns, C. J. and Mandel, J.: A Two-Stage Ensemble Kalman Filter for Smooth Data Assimilation, Environ. Ecol. Stat., 15, 101-110, doi:10.1007/s10651-007-0033-0, 2008.

Kim, M.: Reaction Diffusion Equations and Numerical Wildland Fire Models, Ph.D. thesis, University of Colorado Denver, 2011.

Kochanski, A., Jenkins, M., Krueger, S. K., Mandel, J., Beezley, J. D., and Clements, C. B.: Evaluation of The Fire Plume Dynamics Simulated by WRF-Fire, Presentation at AGU Fall Meeting 2010, available at: http://www.openwfm.org/wiki/File: AGU.2010.kochanski.key.gz (last access: 4 July 2011), 2010.

Kondratenko, V. Y., Beezley, J. D., Kochanski, A. K., and Mandel, J.: Ignition from a Fire Perimeter in a WRF Wildland Fire Model, Paper 9.6, 12th WRF Users' Workshop, National Center for Atmospheric Research, June 20-24, 2011, 2011.

Linn, R., Reisner, J., Colman, J. J., and Winterkamp, J.: Studying wildfire behavior using FIRETEC, Int. J. Wildland Fire, 11, 233246, doi:10.1071/WF02007, 2002.

Litta, A. J. and Mohanty, U. C.: Simulation of a severe thunderstorm event during the field experiment of STORM programme 2006, using WRF-NMM model, Current Science, 95, 204-215, 2008.

Mallet, V., Keyes, D. E., and Fendell, F. E.: Modeling Wildland Fire Propagation with Level Set Methods, Comput. Math. Appl., 57, 1089-1101, doi:10.1016/j.camwa.2008.10.089, 2009.

Mandel, J., Chen, M., Franca, L. P., Johns, C., Puhalskii, A., Coen, J. L., Douglas, C. C., Kremens, R., Vodacek, A., and Zhao, W.: Dynamic Data Driven Wildfire Modeling, UCD CCM Report 208, available at: http://ccm.ucdenver.edu/reports (last access: 4 July 2011), 2004a.

Mandel, J., Chen, M., Franca, L. P., Johns, C., Puhalskii, A., Coen, J. L., Douglas, C. C., Kremens, R., Vodacek, A., and Zhao, W.: A Note on Dynamic Data Driven Wildfire Modeling, in: Computational Science - ICCS 2004, edited by: Bubak, M., van Albada, G. D., Sloot, P. M. A., and Dongarra, J. J., vol. 3038 of Lecture Notes in Computer Science, Springer, 725731 doi:10.1007/b97989, 2004b

Mandel, J., Beezley, J. D., Coen, J. L., and Kim, M.: Data Assimilation for Wildland Fires: Ensemble Kalman filters in coupled atmosphere-surface models, IEEE Control Systems Magazine, 29, 47-65, doi:10.1109/MCS.2009.932224, 2009.

Mandel, J., Beezley, J. D., and Kondratenko, V. Y.: Fast Fourier Transform Ensemble Kalman Filter with Application to a Coupled Atmosphere-Wildland Fire Model, in: Computational Intelligence in Business and Economics, Proceedings of MS'10, edited by: Gil-Lafuente, A. M. and Merigo, J. M., World Scientific, 777-784, 2010.

Mandel, J., Beezley, J. D., and Cobb, L.: Spectral and morphing ensemble Kalman filters, 91st American Meterological Society Annual Meeting, Seattle, WA, January 2011, available at: http://ams.confex.com/ams/91Annual/webprogram/ Paper185877.html (last access: 4 July 2011), 2011.

Mazzoleni, S. and Giannino, F.: Tiger - 2D Fire Prop- agation Simulator Model, http://fireintuition.efi.int/products/ tiger---2d-fire-propagation-simulator-model.fire (last access: December 2010), 2010.

Mell, W., Jenkins, M. A., Gould, J., and Cheney, P.: A physicsbased approach to modelling grassland fires, Intl. J. Wildland Fire, 16, 1-22, doi:10.1071/WF06002, 2007.

Michalakes, J.: RSL: A parallel runtime system library for regional atmospheric models with nesting, in: Structured Adaptive Mesh Refinement (SAMR) Grid Methods, edited by Baden, S. B., Chrisochoides, N. P., Gannon, D. B., and Norman, M. L., Springer, 59-74, 2000.

Morvan, D. and Dupuy, J. L.: Modeling the propagation of a wildfire through a Mediterranean shrub using a multiphase formulation, Combust. Flame, 138, 199-210, doi:10.1016/j.combustflame.2004.05.001, 2004.

Osher, S. and Fedkiw, R.: Level Set Methods and Dynamic Implicit Surfaces, Springer, New York, 2003.

Patton, E. G. and Coen, J. L.: WRF-Fire: A Coupled Atmosphere-Fire Module for WRF, in: Preprints of Joint MM5/Weather Research and Forecasting Model Users' Workshop, Boulder, CO, June 22-25, NCAR, available at: http://www.mmm.ucar.edu/mm5/workshop/ws04/Session9/ Patton_Edward.pdf, 221-223, 2004.

Rothermel, R. C.: A Mathematical Model for Predicting Fire Spread in Wildland Fires, USDA Forest Service Research Paper INT-115, available at: http://www.treesearch.fs.fed.us/pubs/ 32533 (last access: 4 July 2011), 1972.

Scott, J. H. and Burgan, R. E.: Standard Fire Behavior Fuel Models: A Comprehensive Set For Use with Rothermel's Surface Fire Spread Model, Gen. Tech. Rep. RMRS-GTR-153. Fort Collins, CO, US Department of Agriculture, Forest Service, Rocky Mountain Research Station, available at: http://www. fs.fed.us/rm/pubs/rmrs_gtr153.html (last access: 4 July 2011), 2005.

Skamarock, W. C., Klemp, J. B., Dudhia, J., Gill, D. O., Barker, D. M., Duda, M. G., Huang, X.-Y., Wang, W., and Powers, J. G.: A Description of the Advanced Research WRF Version 3, NCAR Technical Note 475, available at: http://www.mmm.ucar. edu/wrf/users/docs/arw_v3.pdf (last access: 4 July 2011), 2008.

Sullivan, A. L.: A review of wildland fire spread modelling, 1990present, 1: Physical and quasi-physical models, 2: Empirical and quasi-empirical models, 3: Mathematical analogues and simulation models, Int. J. Wildland Fire, 18(1), 347-368, (2), 369-386, (3), 387-403, doi:10.1071/WF06143, doi:10.1071/WF06142, doi:10.1071/WF06144, 2009.

Sun, R., Krueger, S. K., Jenkins, M. A., Zulauf, M. A., and Charney, J. J.: The importance of fire-atmosphere coupling and boundarylayer turbulence to wildfire spread, Int. J. Wildland Fire, 18, 50 60, doi:10.1071/WF07072, 2009.

Wang, W., Bruyère, C., Duda, M., Dudhia, J., Gill, D., Lin, H.-C., Michalakes, J., Rizvi, S., Zhang, X., Beezley, J. D., Coen, J. L., and Mandel, J.: ARW Version 3 Modeling System User's Guide, Mesoscale \& Miscroscale Meteorology Division, National Center for Atmospheric Research, available at: http://www.mmm.ucar.edu/wrf/users/docs/user_guide V3/ARWUsersGuideV3.pdf (last access: 4 July 2011), 2010.

WRF Working Group 2: WRF Coding Conventions, available at: http://www.mmm.ucar.edu/wrf/WG2/WRF_conventions.html (last access: April 2007), 2007. 